\title{
GEOCHEMICAL CONTRIBUTIONS OF REE'S BEARING PEGMATITES, WITHIN KADABORA GRANITIC PLUTON, CENTRAL EASTERN DESERT, EGYPT
}

\author{
El Afandy, A. H., Moghazy, N. M. and El Shaib, G. B.
}

Nuclear Materials Authority, Cairo.

\begin{abstract}
Pegmatitic bodies are encountered within the Kadabora granite to which they are spatially and genetically related. These bodies bearing REEs, $\mathrm{U}, \mathrm{Th}, \mathrm{Y}, \mathrm{Zr}, \mathrm{Hf}, \mathrm{Nb}, \mathrm{Ta}$, and $\mathrm{Cs}$. The mineralized pegmatites are characterized by high $\Sigma$ REEs contents ranging from $3404 \mathrm{ppm}$ to 15196 with an average of $7516 \mathrm{ppm}$. The assemblage of the accessory minerals encountered in the mineralized pegmatites are zircon, allanite, fergusonite, uranothorite, betafite, samarskite, pyrochlore, bastnaesite, cerianite, rutile, euxenite and opaques. Each of these minerals is bearing certain trace elements ; zircon ( $\mathrm{Zr}, \mathrm{Hf}, \mathrm{Th})$; fergusonite $(\mathrm{U}, \mathrm{Th}, \mathrm{Zr})$; uranothorite $(\mathrm{Th}, \mathrm{U})$; betafite $(\mathrm{U}, \mathrm{Nb}, \mathrm{Ta})$; samarskite $(\mathrm{Nb}, \mathrm{Ta}, \mathrm{U})$; pyrochlore $(\mathrm{Nb}, \mathrm{Ta})$; bastnaesite (REEs); cerianite (Ce, Th, $\mathrm{Nb}, \mathrm{Ta}, \mathrm{Zr}$ ) and rutile (Ti). The REEs distribution pattern in the mineralized pegmatites reflects the role of these minerals as REEs accumulators e. g. betafite, bastnaesite and cerianite host LREEs while zircon, allanite and samarskite host HREEs.

The REEs pattern is characterized by HREEs enrichment relative to LREEs with convex tetrad effect and a sharp negative Eu anomaly. This well demonstrated pattern reflects highly differentiated rocks associated with self strong hydrothermal interaction. It seems that parent magma has undergone extensive magmatic differentiation, during associated with syngenetic interaction of aqueous hydrothermal fluids resulted in the tetrad effect of REEs distribution forming highly mineralized pegmatites.
\end{abstract}

Keywords: Ree's, pegmatites, kadabora, granite, Central Eastern Desert, Egypt.

\section{INTRODUCTION}

Pegmatites are holocrystalline rocks that are at least appreciably of coarser-grains than the hosting granitic plutonic rocks. Pegmatites often have very irregular forms. Their contacts with the country rocks are seldom sharp and often appear to merge into the hosting rocks surrounding them. Granitic pegmatites commonly form tabular or flatly lenticular minor rock bodies. They range from tens of centimeters to hundreds of meters in length. They are usually found within or at the margins of batholiths and stocks of granites and granodiorites near their apical zones. They predominantly consist of quartz, feldspars and occasionally with or without mica.

The classifications of pegmatites are based mainly on several parameters such as; field aspects, country rock relations, form, size, composition, texture and internal structure. Generally, pegmatites are classified as simple and complex. The simple pegmatites are those having simple mineralogy and no welldeveloped zoning, while the complex pegmatites are those containing assemblages of rare minerals and more than 100 accessory minerals.

Recently, pegmatites were differentiate by their geological tectonic setting, i.e, pegmatites associated with late-, to post-tectonic granitoid magmatism or orogenic environments and pegmatites generated by anorogenic mostly rift related granites of the bimodal gabbro and granite suites, (Cerny, 1982; Norton, 1983; Cerny and Burt, 1984).

The origin of pegmatites has been discussed by many authors, (Procter and El Etr, 1968 and Grinsburg et al., 1979). They showed that the pegmatitic fluids are genetically related to nearby granitic intrusions. Others have attributed their origin to direct anatexic generation of incompatible elements enriched granitic magma (Stewart, 1978). The cooling of granitic magma may produce either aplites or pegmatites, 
El Afandy, et al

depending mainly on the conditions of cooling and the speed with which the volatile components are released from the magma (Stewart, Op. Cit). The large crystals tend to crystallize from an aqueous fluid. However, the complex pegmatites often contain a large number of different mineral species, and it can thus be assumed that they evolved from complex polyphase systems.

According to Jahns and Burnham (1969), the parental magma of most granitic pegmatites crystallizes in three stages: (a) crystallization of essentially anhydrous minerals such as the feldspars and quartz; (b) the crystallization of minerals from both a silicate liquid and a coexisting aqueous fluid of considerably lower viscosity; partitioning of the constituents between the silicate liquid and the aqueous fluid, rapid diffusion of constituents through the aqueous fluid, and the upward movement of the fluid phase, all contribute to the formation of pods and zones of different modal composition and texture; (c) crystallization and metasomatism by aqueous fluids after the crystallization of the silicate phases. The crystallization of most granitic pegmatites is believed to occur in what is essentially a closed system. Complex zoning patterns may develop in some pegmatites because the space hosting the pegmatite continues to open while it is crystallizing. The results are multiple injection, and protracted period of crystallization and contact metasomatism. Both the aplites and pegmatites can be regarded as evidence bearers concerning the nature of the late stage drainage systems that evolved in crystallizing batholiths.

The Arabian-Nubian Shield (ANS) lies at the northern part of the East African Orogen (EAO) or East African Antarctic Orogen (EAAO) and represents the upper crustal equivalent of the high-grade Mozambique Belt (MB). The ANS is considered as the largest tract of juvenile Neoproterozoic continental crust on Earth, and is regarded by others as a major suture zone separating east- and westGondwanalands. It is dominated by Neoproterozoic juvenile arcs, post-amalgamation depositional basins ( $\approx$ forty basins all over the ANS), voluminous granitoid and gabbroic intrusions, and enclaves of preNeoproterozoic crust. This collage was evolved during much debatable late Cryogenian-Ediacaran (650$542 \mathrm{Ma}$ ) depositional, tectonic, metamorphic and intrusive events.

The Arabian-Nubian Shield (ANS) is an accretion-type orogen comprising a stack of thin-skinned nappes resulting from the oblique convergence of bounding plates (Fritz et al. 2013). It constitutes a southward narrowing belt internally structured by individual terranes.

Pegmatites are widely distributed in the northern Arabian Nubian Shield (ANS) in the Eastern Desert of Egypt. Recently, many workers have focused on characterization, geochemistry, mineralization, and radioactivity of some pegmatite occurrences (e.g., El Sheshtawy et al. 1988; Darwish 1991; El-Aassy et al. 1993, 1997; Abu El-Enen 1995; El-Essawy et al. 1997; El-Mowafy et al. 2001; Sherif 2003; Abdel Wahed, et al, 2005, El-Nahas 2012; Draz 2013; Ghazaly et al. 2013 and Abu Steet, 2018). The pegmatites are present in various types of the country rocks and were formed in pre-, syn-, and latetectonic environments (Rashwan 1991). Most of pegmatites are cogenetic related to the younger granites and are generally of granitic composition. Micas, rare earth elements (REEs), beryl, tourmaline, fluorite, vermiculite, corundum, and asbestos-bearing pegmatites were observed (El-Shazly et al. 1975a, b; ElSharkawy and Harraz 2001). The pegmatites in Egypt are mainly quarried for the feldspars and quartz to be used in ceramic and glass industries, respectively.

In the scope of the present work, 23 pegmatitic bodies located in the study area (Fig. 1) have been focused and investigated. Field observations, petrography, mineralogy and radioactivity of these pegmatites were carried out due to their higher radioactivity than the hosting granitic pluton and allover the studied area.

\section{GEOLOGIC SETTING}

Kadabora area lies in the Central Eastern Desert of Egypt between latitudes $25^{\circ} 34^{`}-25^{\circ} 37^{`} \mathrm{~N}$ and longitudes $34^{\circ} 18^{\circ}-34^{\circ} 35^{\circ} \mathrm{E}$ covering about $35 \times 45 \mathrm{~km}$, and forms a roughly circular mass that oblates in $\mathrm{NE}$ direction. The pluton intrudes with sharp contacts the low-grade metavolcano-sedimentary assemblage. The granite is differentiated into: monzoganite core of small aerial extent and peripheral syenogranite with gradational contact (Ahmed, 2004). 


\section{Geochemical contributions of Ree's bearing pegmatites}

Twenty-three sites of pegmatitic bodies occurring as pockets, veins, circular to oblate-shaped intrusions commonly cutting with sharp contact the peripheral syenogranite forming a pegmatite field were encountered in the study area (Fig. 1). They are classified into two types: quartz-feldspar-mica pegmatites type comprising 10 bodies and quartz-feldspar pegmatites type comprising 13 bodies. These pegmatites are mainly concentrated in the Kadabora syenogranites. These sites include; eight at Gabal Abu Dob, two at Gabal Kadabora El Hamra, three along Wadi Kab El Rakab, seven at Gabal Abu Ateila, three at Gabal Klia El Rakab (Fig. 1).

Fig. 1: Geological map of Kadabora grantic mass (after Abdalla and ElAfandy, 2003).

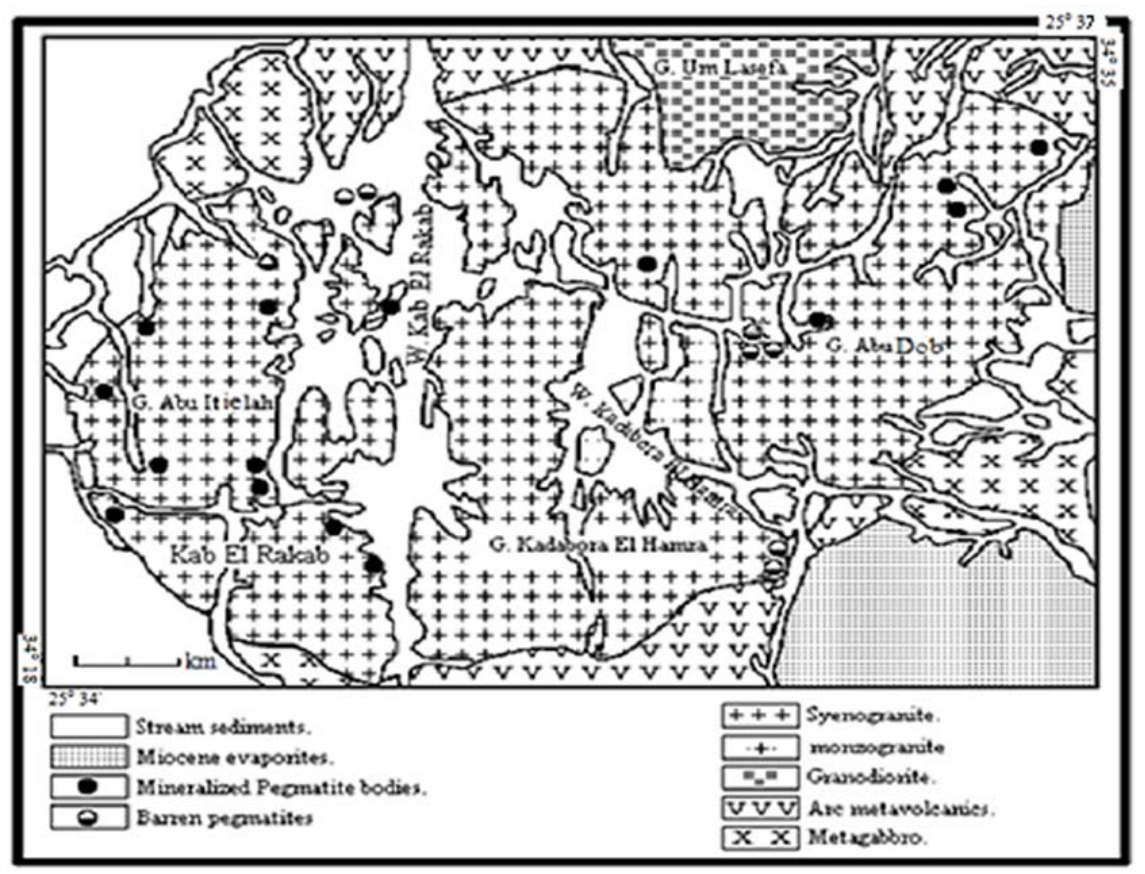

The pegmatite bodies have variable sizes and range from very small as thin veins to rounded and elongated bodies of different shapes. These pegmatites show gradational contacts with the hosting granites and intruded with basic dykes that affected the Kadabora seynogranites (Fig. 2.a). Their size, shap and frequency suggest a case of development in the late stages of the granitic host.

Generally, the pegmatitic bodies in Kadabora pluton have similar mineralogical compositions to the hosting granitic rocks. They are composed mainly of potash feldspars, plagioclases, quartz and micas. According to Brotzen (1959) classification of pegmatites, these pegmatitic bodies represent the simple type. As the ideal simple pegmatite containe feldspars, biotites and quartz, with subordinate hornblende, muscovite and tourmaline, while as the complex pegmatite contains abundant muscovite and lithium minerals such as lithium mica and spodumene.

Gabal Abu Dob and Abu Ateila pegmatites are composed of K-feldspar, quartz, plagioclase and prismatic biotite (1-5 cm in length), (Fig. 2b-e \& Fig.3a-d). Zoned bodies with frequently primary zoned internal structure are occasionally encountered (Abdalla and El Afandy, 2003). They are more or less circular in outcrop with diameters ranging between 5 and $30 \mathrm{~m}$. These pegmatites consist of subhedral Kfeldspar, albite, quartz and prismatic, tabular biotite crystals. The coarse grained pegmatites are characterized by gigantic K-feldspar crystals reaching $20 \mathrm{~cm}$ and either albite or quartz. Zircon, fluorite, allanite, fergusonite, uranothorite, betafite, samarskite, pyrochlore, bastnaesite, cerianite, rutile, euxenite and opaques are accessories (Dawood, 1999; Abdalla and El Afandy, 2003; Ahmed, 2004).

\section{GEOCHEMISTRY}

A total of nineteen whole-rock samples of Kadabora pegmatites were collected and analyzed for major oxides, trace and rare earth elements applying combined techniques of XRF and ICP-MS at Act. Labs, Canada. 
El Afandy, et al

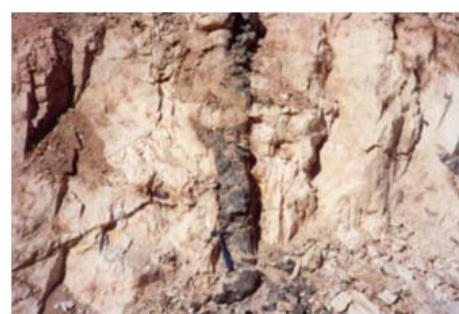

Fig. 2a: Close up view showing vertical basic dyke intruding the pegmatitic body at G. Abu Dob, striking N$\mathrm{S}$, Looking $\mathrm{N}$.

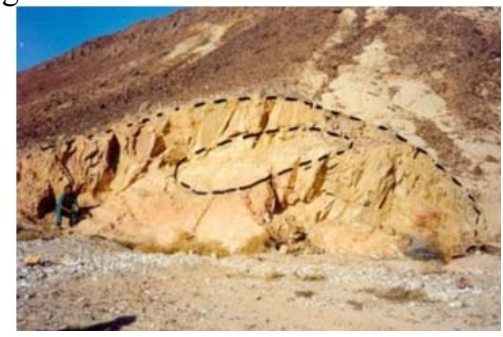

Fig. 2c: Elongated zoned pegmatitic body at W. Kab El Rakab, striking N-S, Looking E.

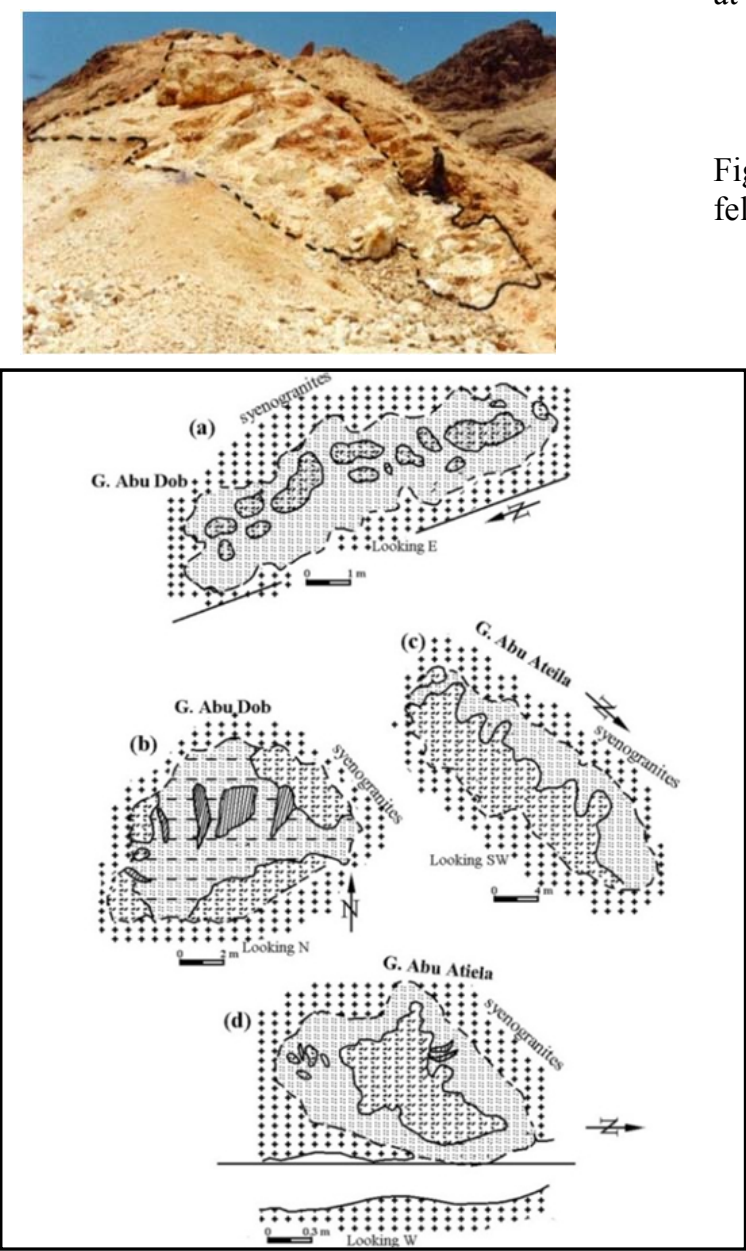

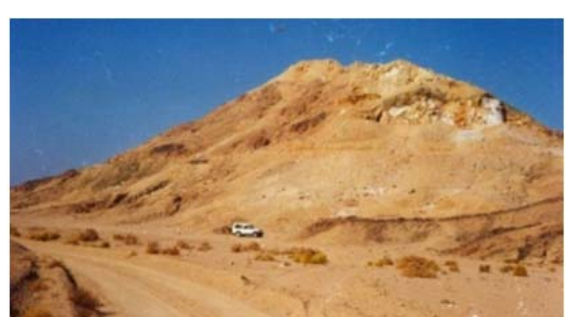

Fig. 2b: Large zoned pegmatite body at G. Abu Dob, slightly elongated to N-S trend (fault related), Looking W.

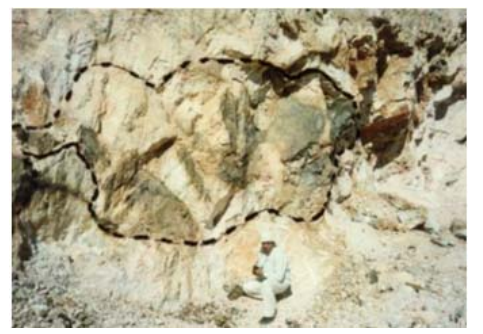

Fig. 2d: Large zoned pegmatitic body, (quartz in the margin, followed by plagioclase and mica in the core) at G. Abu Dob, Looking N.

Fig. 2e: Large zoned pegmatitic body with quartz and feldspars, at G. Abu Ateila, Looking W.

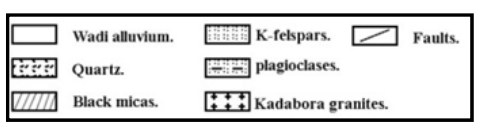

Fig. 3 a, b, c \& d: Sketchs for some zoned pegmatites in Kadabora area.

Major oxides, trace and rare earth elements content are given in Tables $1 \& 2$. The displayed geochemical data shows that the ten mineralized pegmatite samples are distinguished from the normal non-mineralized samples by the extremely high anomalous content of REEs, $\mathrm{U}, \mathrm{Th}, \mathrm{Y}, \mathrm{Zr}, \mathrm{Hf}, \mathrm{Nb}, \mathrm{Ta}$ and 


\section{Geochemical contributions of Ree's bearing pegmatites}

$\mathrm{Cs}$ beside $\mathrm{Au}, \mathrm{Ag}, \mathrm{Pb}$ and occasionally $\mathrm{Cr}$ and $\mathrm{Ni}$. However, the two types show the same major oxides average content except $\mathrm{Al}_{2} \mathrm{O}_{3}, \mathrm{Fe}_{2} \mathrm{O}_{3}, \mathrm{CaO}$ and $\mathrm{Na}_{2} \mathrm{O}$. The mineralized pegmatites contain higher $\mathrm{Al}_{2} \mathrm{O}_{3}$ $(16.7 \%), \mathrm{Fe}_{2} \mathrm{O}_{3}(2.4 \%)$ and $\mathrm{CaO}(0.5 \%)$ and lower $\mathrm{Na}_{2} \mathrm{O}(3.5 \%)$ contents than the non-mineralized ones $(15 \%, 0.7 \%, 0.2 \%$ and $6.8 \%$ respectively). The average concentration of trace elements in the mineralized to the non-mineralized pegmatites (Fig. 4) shows the relative enrichment and depletion as well as the relative mobilities of these elements.

Table 1: Chemical composition of non-mineralized pegmatites.

\begin{tabular}{|c|c|c|c|c|c|c|c|c|c|c|}
\hline Sample & 163 & 164 & 165 & 166 & 176 & 185 & 186 & 187 & 189 & av \\
\hline \multicolumn{11}{|c|}{ Major oxides (\%) } \\
\hline $\mathrm{SiO}_{2}$ & 71.63 & 70.15 & 67.77 & 71.99 & 70.21 & 70.5 & 70.32 & 70.65 & 69.52 & 70.30 \\
\hline $\mathrm{TiO}_{2}$ & 0.02 & 0.02 & 0.02 & 0.02 & 0.02 & 0.33 & 0.01 & 0.02 & 0.02 & 0.05 \\
\hline $\mathrm{Al}_{2} \mathrm{O}_{3}$ & 14.02 & 14.11 & 17.07 & 14.32 & 15.43 & 14.51 & 14.49 & 15.43 & 15.5 & 14.99 \\
\hline $\mathrm{Fe}_{2} \mathrm{O}_{3}$ & 0.36 & 0.48 & 0.19 & 0.56 & 0.99 & 1.23 & 1.37 & 0.54 & 0.39 & 0.68 \\
\hline $\mathrm{MnO}$ & 0.01 & 0.01 & 0.01 & 0.01 & 0.01 & 0.01 & 0.01 & 0.01 & 0.01 & 0.01 \\
\hline $\mathrm{MgO}$ & 0.02 & 0.03 & 0.02 & 0.05 & 0.07 & 0.03 & 0.05 & 0.13 & 0.03 & 0.05 \\
\hline $\mathrm{CaO}$ & 0.17 & 0.11 & 0.18 & 0.2 & 0.22 & 0.4 & 0.43 & 0.25 & 0.11 & 0.23 \\
\hline $\mathrm{Na}_{2} \mathrm{O}$ & 6.87 & 3.76 & 7.67 & 5.58 & 6.32 & 9.4 & 9.46 & 8.91 & 3.34 & 6.81 \\
\hline $\mathrm{K}_{2} 0$ & 6.91 & 10.96 & 7.07 & 5.28 & 4.73 & 2.88 & 2.81 & 3.14 & 10.07 & 5.98 \\
\hline $\mathrm{P}_{2} \mathrm{O}_{5}$ & 0.01 & 0.01 & 0.01 & 0.01 & 0.01 & 0.01 & 0.01 & 0.01 & 0.01 & 0.01 \\
\hline Total & 100.02 & 99.64 & 100.01 & 98.02 & 98.01 & 99.3 & 98.96 & 99.09 & 99 & 99.12 \\
\hline LOI & 0.8 & 0.49 & 0.68 & 0.48 & 0.7 & 0.65 & 0.55 & 0.54 & 0.29 & 0.58 \\
\hline \multicolumn{11}{|c|}{ Trace elements (ppm) } \\
\hline $\mathrm{Cr}$ & 5 & 196 & 245 & 183 & 182 & 258 & 236 & 260 & 177 & 193.56 \\
\hline $\mathrm{Ni}$ & 66 & 69 & 62 & 46 & 68 & 77 & 89 & 56 & 47 & 64.44 \\
\hline Co & 1 & 2 & 1 & 1 & 1 & 2 & 4 & 1 & 1 & 1.56 \\
\hline $\mathrm{Sc}$ & 1 & 1 & 1 & 1 & 1 & 2 & 2 & 1 & 1 & 1.22 \\
\hline V & 2 & 2 & 2 & 2 & 3 & 2 & 3 & 2 & 3 & 2.33 \\
\hline $\mathrm{Cu}$ & 4 & 22 & 5 & 5 & 8 & 41 & 49 & 7 & 19 & 17.78 \\
\hline $\mathrm{Pb}$ & 21 & 17 & 28 & 10 & 10 & 21 & 23 & 9 & 19 & 17.56 \\
\hline $\mathrm{Zn}$ & 12 & 5 & 14 & 2 & 4 & 39 & 47 & 3 & 3 & 14.33 \\
\hline $\mathrm{Cd}$ & 0.4 & 0.45 & 0.38 & 0.47 & 0.5 & 0.6 & 0.43 & 0.48 & 0.49 & 0.47 \\
\hline As & 0.3 & 0.4 & 0.5 & 0.5 & 5.1 & 5.9 & 7.2 & 4.6 & 3.5 & 3.11 \\
\hline $\mathrm{Se}$ & 2 & 3 & 2 & 2 & 3 & 3 & 2 & 2 & 2 & 2.33 \\
\hline $\mathrm{Ag}$ & 0.4 & 0.4 & 0.4 & 0.4 & 0.4 & 0.7 & 0.8 & 0.4 & 0.4 & 0.48 \\
\hline $\mathrm{Au}$ & 1 & 1 & 1 & 1 & 1 & 1 & 1 & 1 & 1 & 1.00 \\
\hline $\mathrm{Rb}$ & 506 & 637 & 580 & 182 & 175 & 151 & 93 & 128 & 651 & 344.78 \\
\hline Cs & 2 & 3 & 2 & 1 & 1 & 1 & 1 & 1 & 3 & 1.67 \\
\hline $\mathrm{Ba}$ & 50 & 46 & 48 & 50 & 42 & 47 & 46 & 48 & 170 & 60.78 \\
\hline $\mathrm{Sr}$ & 7 & 20 & 10 & 11 & 10 & 33 & 36 & 9 & 25 & 17.89 \\
\hline $\mathrm{Ta}$ & 5.8 & 3.9 & 6.4 & 8.1 & 12 & 8.9 & 8.5 & 8.1 & 5.4 & 7.46 \\
\hline $\mathrm{Nb}$ & 80 & 60 & 90 & 125 & 180 & 135 & 130 & 125 & 40 & 107.22 \\
\hline Hf & 7 & 8 & 6 & 7 & 9 & 8 & 10 & 5 & 8 & 7.56 \\
\hline $\mathrm{Zr}$ & 340 & 375 & 337 & 260 & 335 & 300 & 370 & 285 & 340 & 326.89 \\
\hline $\mathrm{Be}$ & 5 & 2 & 5 & 7 & 10 & 5 & 5 & 13 & 2 & 6.00 \\
\hline $\mathrm{U}$ & 8.7 & 4.5 & 5.5 & 5.8 & 9.7 & 14.8 & 20.8 & 5.4 & 3.8 & 8.78 \\
\hline Th & 13.4 & 7.6 & 8.2 & 17.8 & 28 & 19 & 27.1 & 16.7 & 5.4 & 15.91 \\
\hline $\mathrm{Y}$ & 54 & 11 & 44 & 52 & 73 & 28 & 34 & 106 & 49 & 50.11 \\
\hline \multicolumn{11}{|c|}{ Rare earth elements (ppm) } \\
\hline $\mathrm{La}$ & 4.5 & 5.1 & 4.1 & 6 & 9.3 & 3.6 & 4.5 & 13.1 & 3.3 & 5.94 \\
\hline $\mathrm{Ce}$ & 11 & 12 & 10 & 15 & 21 & 10 & 12 & 26 & 8.1 & 13.90 \\
\hline $\operatorname{Pr}$ & 1.5 & 1.7 & 1.5 & 2 & 2.5 & 1.3 & 1.4 & 3.4 & 1.3 & 1.84 \\
\hline $\mathrm{Nd}$ & 7 & 7.4 & 7 & 8.5 & 10 & 7.5 & 7.3 & 15 & 6 & 8.41 \\
\hline $\mathrm{Sm}$ & 1.6 & 1.7 & 1.5 & 1.8 & 2.3 & 1.4 & 1.7 & 3.7 & 1.5 & 1.91 \\
\hline $\mathrm{Eu}$ & 0.2 & 0.2 & 0.2 & 0.2 & 0.2 & 0.2 & 0.2 & 0.2 & 0.2 & 0.20 \\
\hline Gd & 2.1 & 2.3 & 2 & 2.3 & 2.8 & 1.9 & 2 & 3.5 & 2 & 2.32 \\
\hline $\mathrm{Tb}$ & 0.4 & 0.4 & 0.3 & 0.5 & 0.6 & 0.3 & 0.4 & 0.7 & 0.4 & 0.44 \\
\hline Dy & 2.1 & 2.2 & 2.1 & 2.5 & 3.5 & 1.8 & 2 & 4.3 & 1.9 & 2.49 \\
\hline Ho & 0.5 & 0.5 & 0.4 & 0.6 & 0.7 & 0.4 & 0.4 & 0.9 & 0.4 & 0.53 \\
\hline $\mathrm{Er}$ & 1.3 & 1.4 & 1.3 & 1.5 & 1.9 & 1.3 & 1.2 & 2.5 & 1.1 & 1.50 \\
\hline $\mathrm{Tm}$ & 0.2 & 0.2 & 0.2 & 0.2 & 0.3 & 0.2 & 0.2 & 0.4 & 0.2 & 0.23 \\
\hline $\mathrm{Yb}$ & 1.3 & 1.4 & 1.3 & 1.5 & 2 & 1.3 & 1.2 & 2.6 & 1.2 & 1.53 \\
\hline $\mathrm{Lu}$ & 0.2 & 0.2 & 0.2 & 0.3 & 0.3 & 0.2 & 0.2 & 0.4 & 0.2 & 0.24 \\
\hline REEt & 33.9 & 36.7 & 32.1 & 42.9 & 57.4 & 31.4 & 34.7 & 76.7 & 27.8 & 41.51 \\
\hline
\end{tabular}


El Afandy, et al

Table 2: Chemical composition of mineralized pegmatites

\begin{tabular}{|c|c|c|c|c|c|c|c|c|c|c|c|}
\hline Sample & 5 & 7 & 12 & 14 & 32 & 33 & 35 & 37 & 44 & 46 & av \\
\hline \multicolumn{12}{|c|}{ Major oxides (\%) } \\
\hline $\mathrm{SiO}_{2}$ & 69.7 & 67.13 & 70.7 & 70.43 & 68.34 & 66.04 & 59.94 & 69.92 & 70.13 & 67.64 & 68.00 \\
\hline $\mathrm{TiO}_{2}$ & 0.02 & 0.1 & 0.02 & 0.02 & 0.33 & 0.28 & 0.17 & 0.02 & 0.02 & 0.21 & 0.12 \\
\hline $\mathrm{Al}_{2} \mathrm{O}_{3}$ & 17.16 & 17.36 & 15.7 & 15.08 & 16.81 & 18.62 & 17.6 & 15.87 & 15.73 & 17.01 & 16.69 \\
\hline $\mathrm{Fe}_{2} \mathrm{O}_{3}$ & 3.22 & 1.39 & 1.06 & 2.75 & 2.77 & 3.29 & 2.65 & 1.77 & 0.73 & 3.89 & 2.35 \\
\hline $\mathrm{MnO}$ & 0.16 & 0.02 & 0.02 & 0.17 & 0.4 & 0.39 & 0.34 & 0.02 & 0.01 & 0.16 & 0.17 \\
\hline $\mathrm{MgO}$ & 0.25 & 0.32 & 0.03 & 0.27 & 1.13 & 0.98 & 0.65 & 0.42 & 0.05 & 0.75 & 0.49 \\
\hline $\mathrm{CaO}$ & 1.27 & 0.87 & 0.34 & 0.28 & 0.32 & 0.31 & 0.89 & 0.6 & 0.18 & 0.35 & 0.54 \\
\hline $\mathrm{Na}_{2} \mathrm{O}$ & 2.61 & 3.38 & 3.76 & 3.44 & 3.48 & 2.63 & 5.68 & 3.89 & 3.4 & 2.84 & 3.51 \\
\hline $\mathrm{K}_{2} 0$ & 5.42 & 9.4 & 6.76 & 5.42 & 4.78 & 5.75 & 9.95 & 5.55 & 9.75 & 5.5 & 6.83 \\
\hline $\mathrm{P}_{2} \mathrm{O}_{5}$ & 0.13 & 0.03 & 0.06 & 0.14 & 0.08 & 0.11 & 0.12 & 0.06 & 0.01 & 0.09 & 0.08 \\
\hline Total & 99.94 & 100 & 98.45 & 98 & 98.44 & 98.4 & 97.99 & 98.12 & 100.01 & 98.44 & 98.78 \\
\hline LOT & 0.25 & 0.42 & 0.61 & 0.57 & 0.59 & 0.62 & 0.28 & 0.49 & 0.46 & 0.57 & 0.49 \\
\hline \multicolumn{12}{|c|}{ Trace elements (ppm) } \\
\hline $\mathrm{Cr}$ & 4 & 187 & 234 & 4 & 5 & 3 & 5 & 4 & 5 & 127 & 57.80 \\
\hline $\mathrm{Ni}$ & 83 & 61 & 124 & 80 & 8 & 6 & 11 & 98 & 39 & 17 & 52.70 \\
\hline Co & 4 & 4 & 24 & 8 & 9 & 9 & 4 & 8 & 3 & 1 & 7.40 \\
\hline $\mathrm{Sc}$ & 9 & 1 & 19 & 10 & 71 & 71 & 47 & 25 & 1 & 52 & 30.60 \\
\hline V & 26 & 10 & 4 & 29 & 6 & 5 & 12 & 2 & 2 & 16 & 11.20 \\
\hline $\mathrm{Cu}$ & 29 & 16 & 31 & 28 & 11 & 10 & 6 & 25 & 10 & 7 & 17.30 \\
\hline $\mathrm{Pb}$ & 249 & 93 & 230 & 247 & 71 & 74 & 81 & 179 & 99 & 68 & 139.10 \\
\hline $\mathrm{Zn}$ & 157 & 28 & 37 & 155 & 2232 & 2183 & 460 & 23 & 12 & 517 & 580.40 \\
\hline $\mathrm{Cd}$ & 0.9 & 1 & 2.9 & 1.6 & 2.50 & 2.8 & 0.6 & 2.1 & 1.1 & 0.5 & 1.50 \\
\hline As & 4.5 & 1.1 & 5.8 & 3.7 & 3.2 & 3.3 & 5.6 & 5.9 & 1.4 & 1.9 & 3.64 \\
\hline $\mathrm{Se}$ & 54 & 2 & 75 & 29 & 10 & 15 & 8 & 98 & 25 & 9 & 32.50 \\
\hline $\mathrm{Ag}$ & 48 & 49 & 46 & 45 & 48 & 42 & 49 & 46 & 47 & 42 & 46.20 \\
\hline $\mathrm{AU}$ & 32 & 6 & 39 & 24 & 20 & 21 & 39 & 47 & 12 & 12 & 25.20 \\
\hline $\mathrm{Rb}$ & 400 & 567 & 640 & 520 & 764 & 921 & 782 & 440 & 641 & 800 & 647.50 \\
\hline Cs & 4 & 3 & 9 & 8 & 35 & 44 & 33 & 2 & 5 & 37 & 18.00 \\
\hline $\mathrm{Ba}$ & 300 & 50 & 450 & 260 & 200 & 360 & 150 & 400 & 49 & 130 & 234.90 \\
\hline $\mathrm{Sr}$ & 102 & 40 & 86 & 114 & 46 & 53 & 42 & 57 & 19 & 35 & 59.40 \\
\hline $\mathrm{Ta}$ & 121 & 223 & 2110 & 114 & 576 & 604 & 488 & 1460 & 210 & 505 & 641.10 \\
\hline $\mathrm{Nb}$ & 1815 & 3345 & 10650 & 1710 & 8640 & 9060 & 7320 & 9900 & 3150 & 7575 & 6316.50 \\
\hline $\mathrm{Hf}$ & 2860 & 665 & 4140 & 2670 & 1330 & 1820 & 1570 & 3320 & 590 & 1350 & 2031.50 \\
\hline $\mathrm{Zr}$ & 10582 & 2405 & 15318 & 9879 & 4921 & 6734 & 5809 & 12284 & 3404 & 4995 & 7633.10 \\
\hline $\mathrm{Be}$ & 27 & 258 & 233 & 27 & 26 & 31 & 42 & 220 & 258 & 36 & 115.80 \\
\hline $\mathrm{U}$ & 1530 & 192 & 2520 & 1500 & 1650 & 2220 & 690 & 2170 & 193 & 624 & 1328.90 \\
\hline Th & 3430 & 600 & 4340 & 3770 & 2190 & 2870 & 1430 & 3590 & 611 & 1180 & 2401.10 \\
\hline Y & 2792 & 2233 & 5391 & 3043 & 1928 & 3069 & 1555 & 4644 & 2265 & 1065 & 2798.50 \\
\hline \multicolumn{12}{|c|}{ Rare earth elements (ppm) } \\
\hline $\mathrm{La}$ & 1750 & 199 & 536 & 1570 & 154 & 149 & 244 & 359 & 196 & 228 & 538.50 \\
\hline $\mathrm{Ce}$ & 7210 & 1110 & 2300 & 7040 & 578 & 574 & 838 & 1390 & 1060 & 849 & 2294.90 \\
\hline $\operatorname{Pr}$ & 650 & 125 & 370 & 630 & 82 & 79 & 120 & 210 & 170 & 115 & 255.10 \\
\hline $\mathrm{Nd}$ & 1120 & 522 & 1480 & 845 & 293 & 227 & 285 & 880 & 691 & 392 & 673.50 \\
\hline $\mathrm{Sm}$ & 194 & 134 & 485 & 195 & 85.3 & 81 & 105 & 380 & 292 & 98 & 204.93 \\
\hline $\mathrm{Eu}$ & 6.4 & 2 & 12.7 & 5.3 & 1.7 & 1.6 & 1.8 & 1.9 & 1.9 & 1.5 & 3.68 \\
\hline Gd & 235 & 125 & 430 & 230 & 80 & 90 & 80 & 280 & 250 & 78 & 187.80 \\
\hline $\mathrm{Tb}$ & 65 & 30 & 120 & 63 & 30 & 40 & 29 & 94 & 65 & 26 & 56.20 \\
\hline Dy & 451 & 140 & 790 & 435 & 325 & 375 & 310 & 650 & 450 & 290 & 421.60 \\
\hline Ho & 160 & 50 & 230 & 155 & 145 & 165 & 135 & 190 & 120 & 120 & 147.00 \\
\hline $\mathrm{Er}$ & 560 & 165 & 850 & 540 & 520 & 550 & 490 & 720 & 390 & 450 & 523.50 \\
\hline $\mathrm{Tm}$ & 180 & 60 & 330 & 175 & 140 & 170 & 130 & 210 & 75 & 125 & 159.50 \\
\hline $\mathrm{Yb}$ & 2220 & 646 & 3170 & 1990 & 1500 & 2290 & 1180 & 2690 & 706 & 1120 & 1751.20 \\
\hline $\mathrm{Lu}$ & 395 & 95.5 & 580 & 320 & 267 & 362 & 220 & 451 & 105 & 189 & 298.45 \\
\hline REEt & 15196.4 & 3403.5 & 11683.7 & 14193.3 & 4201 & 5153.6 & 4167.8 & 8505.9 & 4571.9 & 4081.5 & 7515.86 \\
\hline
\end{tabular}


Geochemical contributions of Ree's bearing pegmatites
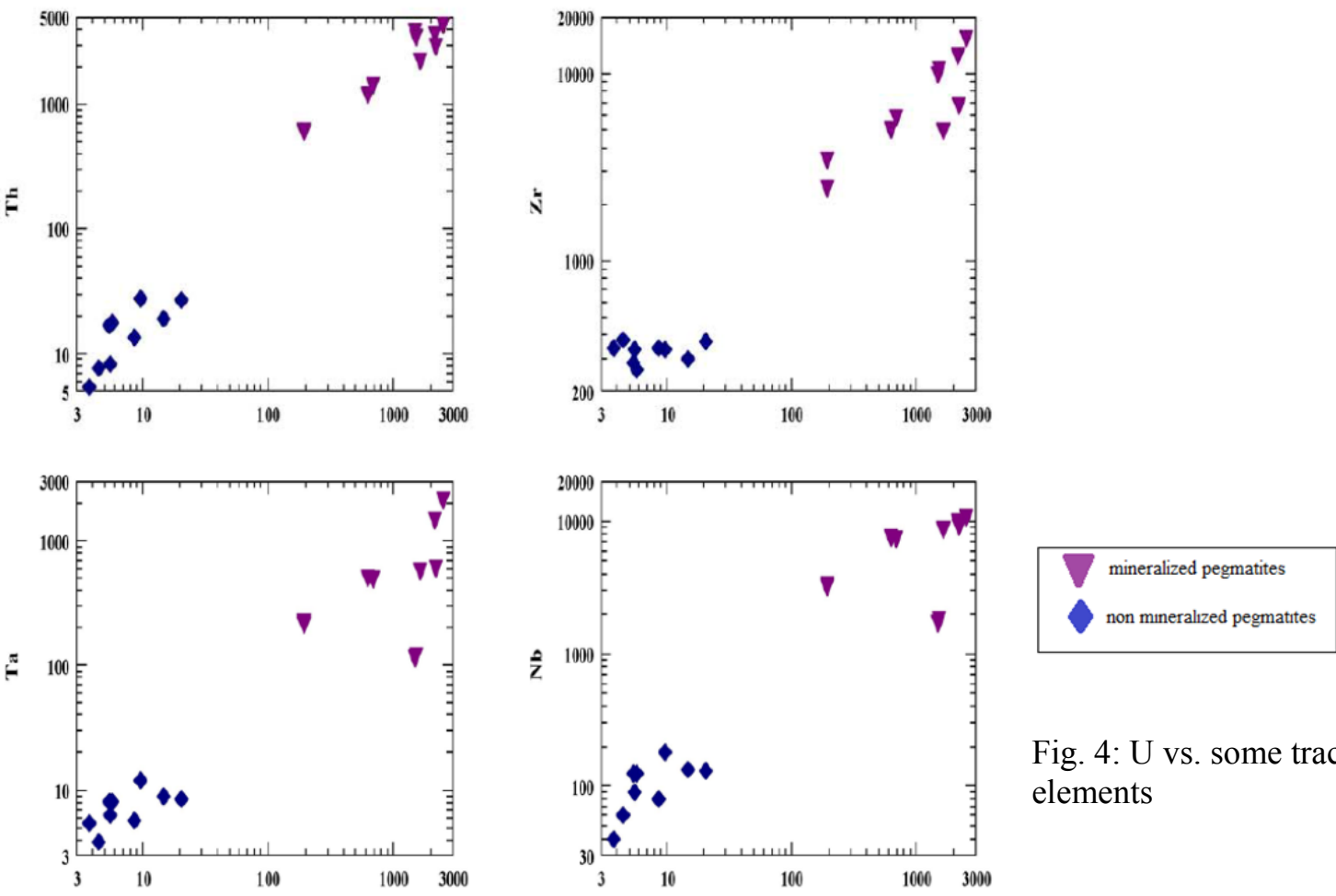

Fig. 4: U vs. some trace elements
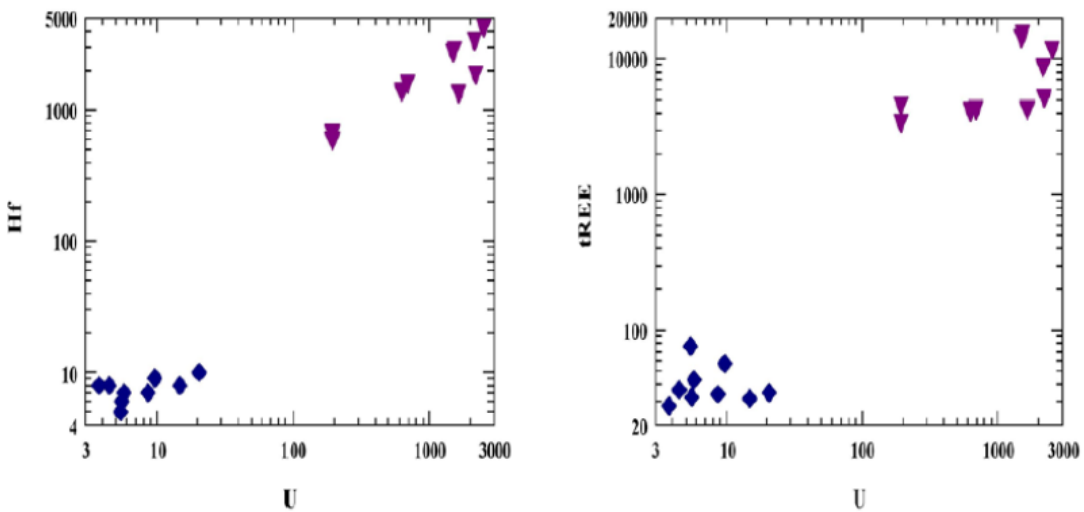

\section{Radioactivity}

The distribution of the radioactive and associated minerals was basically based on the microscopic investigations, XRD analysis and environmental scanning electron microscope (ESEM-XL30) of individual separated minerals. This was carried out on the separated minerals from the bulk samples, having high contents of uranium and/or thorium, using heavy liquids and magnetic fractionations of the heavy minerals using Frantz isodynamic separator. The magnetic fractions were then purified using methylene iodide and clercii solution and finally by hand picking. The purified minerals were then identified by XRD and ESEM techniques. These identified radioactive bearing minerals are zircon, fergusonite, uranothorite, betafite, and samarskilte (Tables 3, 4 and 5, and Figs. 5 and 6).

In igneous rocks, uranium and thorium tend to concentrate in resistate, accessory minerals such as zircon, apatite, sphene, monazite, and allanite (Rogers and Adams, 1969). During fractionation, uranium may become enriched in final fraction of magma, and the residual hydrothermal solution may form uranium rich veins (Goldschmidt, 1954). The redistribution of uranium within the rock is controlled mainly by the hydrothermal processes operative in the granite during the final stages of the crystallization of the granitic magma (O'Connor et al., 1982). 
El Afandy, et al

Table 3: X-ray diffraction pattern of zircon, fergusonite and uranothorite from the studied area.

\begin{tabular}{|c|c|c|c|c|c|c|c|}
\hline \multicolumn{2}{|c|}{ Sample } & \multicolumn{2}{|l|}{ Zircon } & \multicolumn{2}{|c|}{ Fergusonite } & \multicolumn{2}{|c|}{ Uranothorite } \\
\hline DA & $\mathrm{I} / \mathrm{I}^{\mathrm{O}}$ & DA & $\mathrm{I} / \mathrm{I}^{\mathrm{o}}$ & DA & $\mathrm{I} / \mathrm{I}^{\mathrm{o}}$ & DA & $\mathrm{I} / \mathrm{I}^{\mathrm{O}}$ \\
\hline 4.71 & 5 & 4.43 & 45 & & & 4.69 & \\
\hline 4.44 & 27 & & & & & & \\
\hline 4.19 & 2 & & & & & & \\
\hline 3.56 & 9 & 3.30 & 100 & & & 3.50 & 100 \\
\hline 3.31 & 100 & & & 3.12 & 100 & & \\
\hline 3.13 & 10 & & & 3.01 & 20 & & \\
\hline 3.01 & 13 & & & 2.96 & 90 & & \\
\hline 2.97 & 7 & & & & & & \\
\hline 2.92 & 4 & & & & & & \\
\hline 2.84 & 4 & & & & & 2.82 & 40 \\
\hline 2.79 & 3 & & & & & & \\
\hline 2.75 & 3 & & & 2.74 & 40 & & \\
\hline 2.65 & 7 & 2.65 & 8 & 2.64 & 20 & 2.653 & 60 \\
\hline 2.60 & 3 & & & 2.59 & 4 & & \\
\hline 2.52 & 24 & 2.518 & 45 & 2.52 & 4 & 2.499 & 30 \\
\hline 2.34 & 5 & 2.336 & 10 & & & 2.336 & 50 \\
\hline 2.22 & 5 & 2.217 & 8 & 2.22 & 50 & 2.20 & 40 \\
\hline 2.07 & 13 & 2.066 & 20 & & & & \\
\hline 1.91 & 7 & 1.908 & 14 & 1.901 & 30 & 1.869 & 30 \\
\hline 1.84 & 4 & & & 1.855 & 10 & & \\
\hline 1.76 & 6 & 1.75 & 12 & 1.754 & & 1.767 & 10 \\
\hline 1.72 & 19 & 1.712 & 40 & & & 1.47 & 10 \\
\hline 1.66 & 11 & 1.65 & 14 & 1.646 & 10 & 1.653 & 10 \\
\hline 1.57 & 3 & 1.547 & 4 & 1.569 & 10 & & \\
\hline 1.48 & 4 & 1.477 & 8 & 1.478 & 4 & 1.469 & 20 \\
\hline
\end{tabular}

Table 4: X-ray diffraction pattern of zircon, uranothorite and betafite from the studied area.

\begin{tabular}{|c|c|c|c|c|c|c|c|}
\hline \multicolumn{2}{|c|}{ Sample } & \multicolumn{2}{|c|}{ Zircon } & \multicolumn{2}{|c|}{ Uranothorite } & \multicolumn{2}{|c|}{ Betafite } \\
\hline DA & $\mathrm{I} / \mathrm{I}^{\mathrm{o}}$ & DA & $\mathrm{I} / \mathrm{I}^{\mathrm{O}}$ & DA & $\mathrm{I} / \mathrm{I}^{\mathrm{O}}$ & DA & $\mathrm{I} / \mathrm{I}^{\mathrm{O}}$ \\
\hline 4.69 & 2 & & & 4.69 & 90 & & \\
\hline 4.43 & 2.2 & 4.43 & 45 & & & & \\
\hline 4.09 & 2 & & & & & 4.05 & 60 \\
\hline 3.86 & 2 & & & & & & \\
\hline 3.67 & 2 & & & & & & \\
\hline 3.53 & 6 & & & 3.53 & 100 & 3.55 & 20 \\
\hline 3.40 & 4 & & & & & & \\
\hline 3.31 & 100 & 3.30 & 100 & & & & \\
\hline 3.18 & 4 & & & & & 3.20 & 100 \\
\hline 3.06 & 5 & & & & & & \\
\hline 3.01 & 12 & & & & & 2.99 & 10 \\
\hline 2.80 & 7 & & & 2.82 & 40 & 2.81 & 80 \\
\hline 2.74 & 5 & & & & & & \\
\hline 2.69 & 6 & & & & & & \\
\hline 2.66 & 9 & & & 2.065 & 60 & & \\
\hline 2.60 & 4 & 2.65 & 8 & & & 2.61 & 10 \\
\hline 2.52 & 42 & & & 2.499 & 30 & 2.50 & 80 \\
\hline 2.34 & 10 & 2.518 & 45 & 2.336 & 5 & & \\
\hline 2.22 & 6 & 2.336 & 10 & 2.203 & 40 & & \\
\hline 2.07 & 18 & 2.217 & 8 & & & & \\
\hline 1.91 & 10 & 2.066 & 20 & & & & \\
\hline 1.84 & 6 & 1.908 & 14 & 1.869 & 30 & 1.83 & 50 \\
\hline 1.82 & 3 & & & 1.818 & 60 & & \\
\hline 1.76 & 12 & 1.751 & 12 & 1.767 & 10 & & \\
\hline 1.72 & 41 & 1.712 & 40 & 1.074 & 10 & & \\
\hline 1.71 & 32 & & & & & 1.71 & 30 \\
\hline 1.65 & 22 & 1.651 & 14 & 1.65 & 10 & 1.67 & 30 \\
\hline 1.57 & 4 & & & 1.582 & 10 & 1.59 & 10 \\
\hline 1.55 & 3 & 1.547 & 4 & 1.56 & 10 & & \\
\hline 1.48 & 9 & 1.477 & 8 & 1.469 & 20 & 1.48 & 20 \\
\hline
\end{tabular}




\section{Geochemical contributions of Ree's bearing pegmatites}

Tabl 5: X-ray diffraction pattern of fergusonite, zircon and samarskite from the studied area.

\begin{tabular}{|c|c|c|c|c|c|c|c|}
\hline \multicolumn{2}{|c|}{ Sample } & \multicolumn{2}{|c|}{ Fergusonite } & \multicolumn{2}{|l|}{ Zircon } & \multicolumn{2}{|c|}{ Samarskite } \\
\hline DA & $\mathrm{I} / \mathrm{I}^{\mathrm{o}}$ & DA & $\mathrm{I} / \mathrm{I}^{\mathrm{o}}$ & DA & $\mathrm{I} / \mathrm{I}^{\mathrm{o}}$ & DA & $\mathrm{I} / \mathrm{I}^{\mathrm{O}}$ \\
\hline 4.01 & 6 & & & & & & \\
\hline 4.68 & 5 & & & & & & \\
\hline 4.42 & 21 & & & 4.43 & 45 & & \\
\hline 3.56 & 11 & & & & & & \\
\hline 3.30 & 61 & 3.29 & 4 & & & 3.59 & 20 \\
\hline 3.12 & 100 & 3.12 & 100 & 3.30 & 100 & & \\
\hline 3.00 & 96 & 3.01 & 20 & & & 3.13 & 40 \\
\hline 2.96 & 65 & 2.96 & 90 & & & 2.98 & 100 \\
\hline 2.91 & 46 & & & & & & \\
\hline 2.76 & 24 & & & & & 2.92 & 90 \\
\hline 2.74 & 21 & 2.74 & 40 & & & 2.75 & 10 \\
\hline 2.64 & 19 & 2.64 & 20 & 2.65 & 8 & & \\
\hline 2.60 & 20 & 2.59 & 4 & & & 2.52 & 20 \\
\hline 2.52 & 26 & 2.52 & 10 & 2.218 & 45 & & \\
\hline 2.33 & 5 & & & 233 & 10 & & \\
\hline 2.22 & 6 & 2.22 & 4 & 2.217 & 8 & & \\
\hline 2.17 & 6 & 2.16 & 8 & & & 2.17 & 6 \\
\hline 2.07 & 7 & & & 2.066 & 20 & 2.07 & 4 \\
\hline 1.90 & 33 & 1.90 & 50 & 1.908 & 14 & 1.909 & 20 \\
\hline 2.85 & 17 & 1.855 & 30 & & & 1.86 & 20 \\
\hline 1.84 & 20 & & & & & & \\
\hline 1.79 & 3 & & & & & & \\
\hline 1.76 & 11 & 1.754 & 10 & 1.751 & 12 & 1.709 & 20 \\
\hline 1.71 & 14 & & & 1.712 & 40 & 1.65 & 6 \\
\hline 1.65 & 12 & 1.646 & 10 & 1.651 & 14 & & \\
\hline 1.62 & 11 & 1.62 & 10 & & & & \\
\hline 1.60 & 5 & & & & & & \\
\hline 1.57 & 25 & 1.569 & 10 & & & 1.57 & 10 \\
\hline 1.51 & 7 & 1.508 & 6 & & & 1.51 & 10 \\
\hline 1.48 & 97 & 1.478 & 4 & 1.477 & 8 & & \\
\hline
\end{tabular}

Depending on the fO2 oxygen fugacity, uranium also tend to form the relatively soluble uranyl ion $\left(\mathrm{UO}_{2}\right)^{++}$and mineralization may therefore arise through weathering and leaching of the host rock under oxidizing conditions with consequent removal of uranium and deposition elsewhere in fractures and faults (Gabelman, 1970). Uranium may also precipitate in intergranular spaces or is located in the late stage alterations, ( $\mathrm{O}^{\circ}$ Connor op cit). Remobilization of uranium at labile sites could be takes place dairly readily under the action of effective transport fluids such as water removing along channels (e.g. microfractures) in the rock.

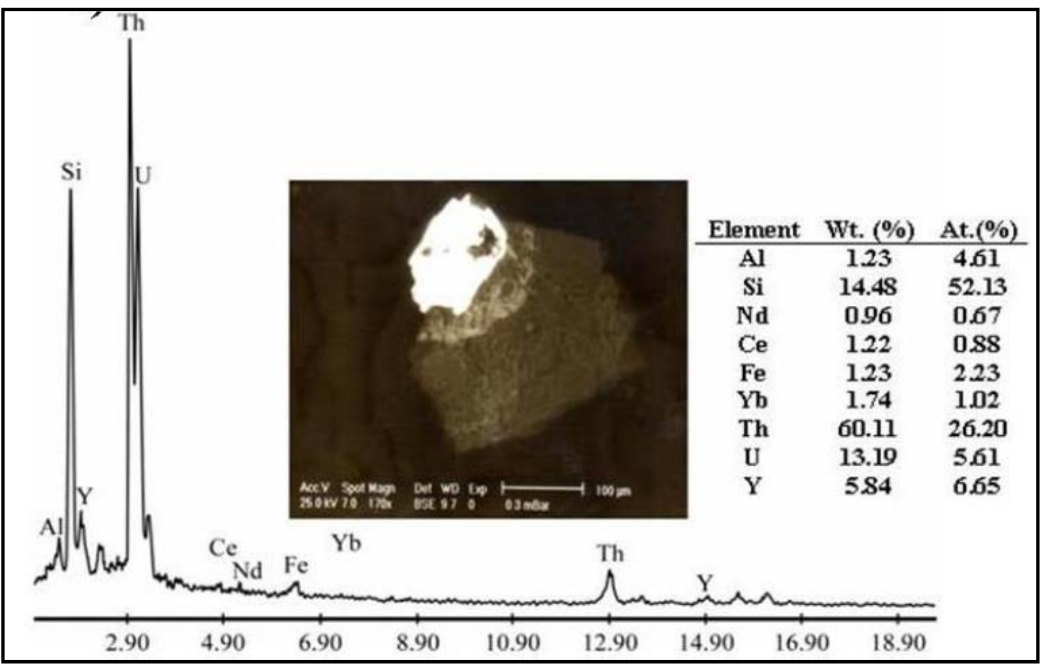

Fig. 5: EDAX pattern and BSE photomicrograph of uranothorite, Kadabora area, SED, Egypt. 
Fig. 6: EDX and back scattered of samarskite in the studied mineralized pegmatite.
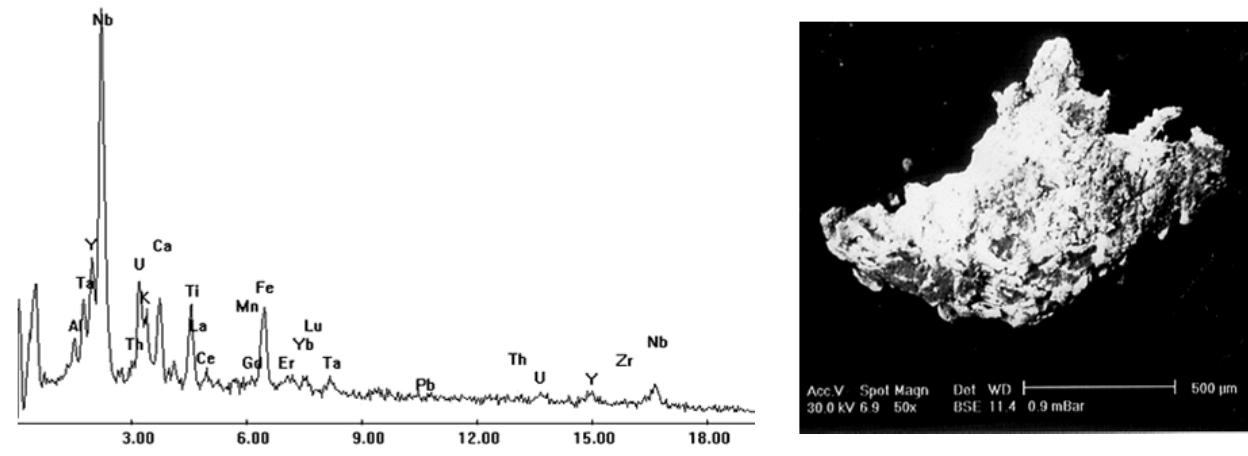

The non-mineralized pegmatites characterized by low contents of $U$ (3.8-20.8ppm) and Th (5.4-28 $\mathrm{ppm}$ ), with $\mathrm{Th} / \mathrm{U}$ ratio in the range from 1.3 to 3.1 and 1.8 in average, while the mineralized pegmatites are characterized by high contents of $\mathrm{U}(192-2520 \mathrm{ppm})$ and $\mathrm{Th}(600-4340 \mathrm{ppm})$, with $\mathrm{Th} / \mathrm{U}$ ratio in the range from 1.3 to 3.2 and 1.8 in average. The mineralized pegmatites are enriched in $U$ and $T h$ by an enrichment ratio of more than 180 , compared to the non-mineralized pegmatites.

Generally, during magmatic differentiation $\mathrm{U}$ and $\mathrm{Th}$ increase but the $\mathrm{Th} / \mathrm{U}$ ratio remains constant. The $\mathrm{U}$ and $\mathrm{Th}$ are generally enriched in the co-magmatic suites of igneous rocks, which are the youngest most silicic and most potassic members (Rogers \& Adams, 1969). The constant Th/U ratio in both mineralized and non-mineralized pegmatites and the positive correlation between $\mathrm{U}$ and $\mathrm{Th}, \mathrm{Zr}, \mathrm{Ta}, \mathrm{Nb}$, $\mathrm{Hf}$ and LREEs in the studied pegmatites (Fig. 4) reflects that the enrichment of uranium and thorium is related to magmatic (hypogene) processes.

Also, the enrichment of both uranium and/or thorium bearing minerals (zircon, fergusonite, uranothorite, betafite, and samarskilte) at the residual magma (pegmatite) indicate that, the enrichment is due to magmatic processes.

\section{Rare earth elements}

The non-mineralized pegmatites are characterized by $\Sigma$ REEs contents ranging from $28 \mathrm{ppm}$ to $77 \mathrm{ppm}$ with an average of $41.6 \mathrm{ppm}$ which are lower than the average REE values $(250 \mathrm{ppm})$ of granitic rocks as given by Herrmann (1970), while the mineralized pegmatites are highly enriched in $\Sigma$ REEs contents than the granitic rocks and their contents are ranging from $3404 \mathrm{ppm}$ to 15196 with an average of $7516 \mathrm{ppm}$. The enrichement ratio compared to the non-mineralized is 1 to 181 .

Chondrite normalized REE pattern (Sun and McDonough, 1989) of the considered pegmatites (Fig. 7) display a wing shape. The non-mineralized pegmatites chondrite-normalized REEs patterns show a relative enrichment in the LREEs compared to the HREEs, with negative Eu-anomaly. The LREEs are fractionated while the HREEs are flat.
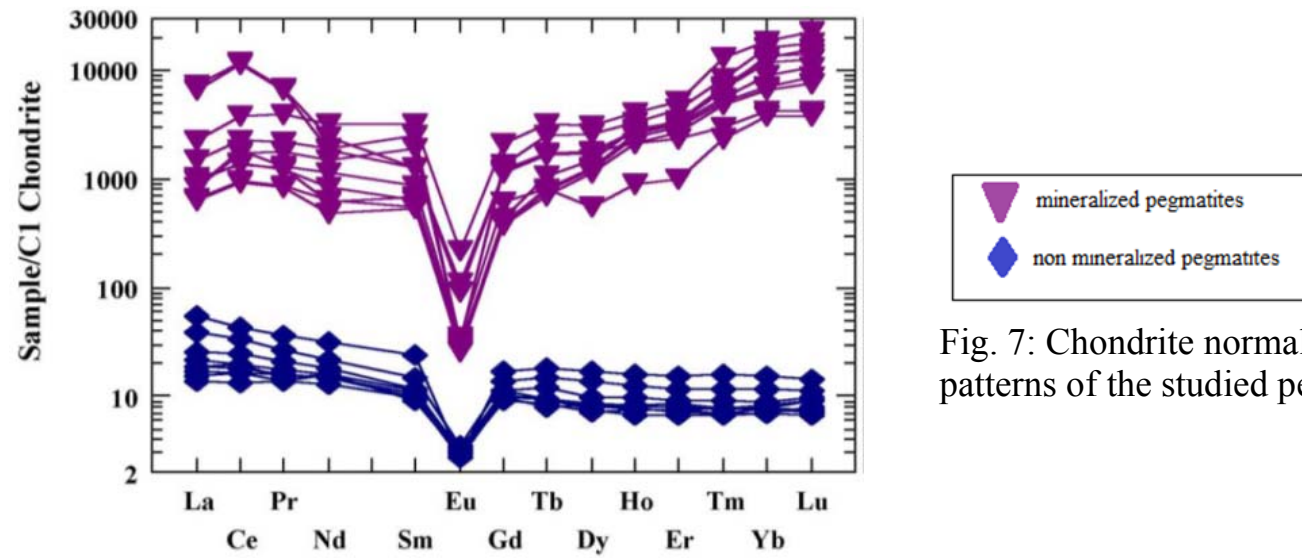

Fig. 7: Chondrite normalized REEs patterns of the studied pegmatites. 


\section{Geochemical contributions of Ree's bearing pegmatites}

The REEs pattern of the mineralized pegmatites is characterized by HREEs enrichment relative to LREEs with (LREE/HREE) $)_{\mathrm{N}}$ ratio ranging from 0.09 to 0.69 . It exhibits sharp negative Eu anomaly with $\left(\mathrm{Eu} / \mathrm{Eu}^{*}\right)$ ranging from 0.02 to 0.09 .

The negative Eu anomaly indicates its depletion in the medium. The late magmatic melt is diagnostically defict in $\mathrm{Eu}$. Eu anomalies (Eu/Eu*) are commonly explained by feldspar fractionation (e.g. Möller and Muecke, 1984). Common mineral fractionation is able to account for $\mathrm{Eu} / \mathrm{Eu}^{*}$ down to a minimum of 0.06 . Ratios $<0.06$ were only achieved in the unrealistic case that feldspar is the exclusive fractionating mineral phase (Irber, 1999). The strong Eu depletion in the late-stage of granitic crystallization may indicate a preferential $\mathrm{Eu}$ fractionation into a co-existing aqueous fluid phase rather than into feldspar (Muecke and Clarke, 1981). This is most probably the case in the studied pegmatites.

\section{Tetrad Effect}

The term tetrad effect is refered to the split of chondrite-normalized REEs patterns into four rounded segments called tetrads (first tetrad, La-Nd, second tetrad $\left(\mathrm{P}_{\mathrm{m}}\right)-\mathrm{Gd}$, third tetrad, Gd-Ho, fourth tetrad, Er$\mathrm{Lu}$ ). The rounded segments are either convex or concave and form M-shaped and $\mathrm{W}$-shaped lanthanide distribution patterns (Masuda et al., 1987). Tetrad effect-like REEs patterns are reported both in magmatic rocks and precipitates from hydrothermal fluids. Recent discussions about the tetrad effect focus on highly evolved igneous rocks (Bau, 1996). Ireber (1999) and Monecke et al., (2002) introduced procedures for quantification of tetrad effect and individual tetrads.

The tetrad effect in lanthanide patterns of whole-rock samples was quantified by equations. 1, 2 and 3 proposed by Irber (1999). The calculated sizes $T_{1}, T_{3}$ and $T$ of the tetrad effect are listed in Table 6.

Table 6: Calculated sizes $\mathrm{T}_{1}, \mathrm{~T}_{3}$ and $\mathrm{T}$ of the tetrad effect

\begin{tabular}{|c|c|c|c|c|}
\hline & sample & $\mathrm{T} 1$ & T3 & $\mathrm{T}$ \\
\hline \multirow{9}{*}{ 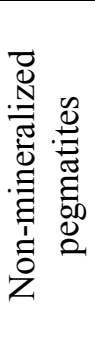 } & 163 & 1.02 & 0.97 & 1.00 \\
\hline & 164 & 1.04 & 0.95 & 0.99 \\
\hline & 165 & 1.02 & 1.25 & 1.13 \\
\hline & 166 & 1.08 & 1.03 & 1.06 \\
\hline & 176 & 1.06 & 1.13 & 1.09 \\
\hline & 185 & 0.98 & 0.92 & 0.95 \\
\hline & 186 & 1.01 & 1.09 & 1.05 \\
\hline & 187 & 0.95 & 1.06 & 1.00 \\
\hline & 189 & 1.03 & 1.06 & 1.04 \\
\hline \multirow{10}{*}{ 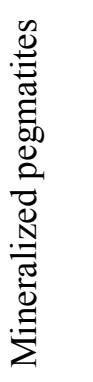 } & 5 & 2.18 & 0.96 & 1.45 \\
\hline & 7 & 1.63 & 0.89 & 1.21 \\
\hline & 12 & 1.46 & 1.06 & 1.25 \\
\hline & 14 & 2.58 & 0.95 & 1.57 \\
\hline & 32 & 1.45 & 1.00 & 1.20 \\
\hline & 33 & 1.63 & 1.09 & 1.34 \\
\hline & 35 & 1.70 & 0.99 & 1.30 \\
\hline & 37 & 1.36 & 1.17 & 1.26 \\
\hline & 44 & 1.63 & 1.07 & 1.32 \\
\hline & 46 & 1.47 & 0.98 & 1.20 \\
\hline
\end{tabular}

The non-mineralized pegmatite samples show smooth $R_{E} E_{s}$ patterns (Fig. 7). The calculated sizes $T_{1}$, $\mathrm{T}_{3}$ and $\mathrm{T}$ of the tetrad effect are below the level of significance (1.1) except in one sample where it is closed to the level of significance. The mineralized pegmatites show a pattern with convex tetrad effect. This pattern is well demonstrated in highly differentiated rocks with strong hydrothermal interaction (Jahn et al., 2001). The tetrad effect values range from 1.2 to 1.57 . The individual tetrads in the chondritenormalized REEs patterns have variable sizes. The first tetrad have the size $\left(\mathrm{T}_{1}=1.36-2.58\right)$ being strongly developed than the third segment $\left(\mathrm{T}_{3}=0.89-1.17\right)$.

The late stage fluid-melt interaction possibly affected the size of the tetrad effect of individual tetrads and may have influenced the anomalous enrichment of different elements in the mineralized pegmatites (Jahn, et al., 2001). 
El Afandy, et al

The convex tetrad effect in granite pattern has been attributed to fractional crystallization during granite differentiation, to a characteristic magma-fluid systems that develop during or before granite crystallization or inherited from an external fluid during or after the emplacement of the magma (Cocherie et al., 1991, MacLennan, 1994, Kawabe, 1995, Irber, 1999 and Monecke et al., 2002). Irber (1999) argued that the convex tetrad effect of granite samples must have resulted from interaction of the granitic melt with a coexisting fluid at a late stage of the granite crystallization after the magma-fluid system was split into magma and fluid subsystems. This implies that the convex tetrad effect in the mineralized pegmatites represents a residual lanthanide pattern (Kawabe, 1995 and Irber, 1999).

Trace elements distribution in common magmatic systems is largely charge and radius controlled. High-field strength elements (HFSE) such as $\mathrm{Y}, \mathrm{REE}, \mathrm{Zr}, \mathrm{Hf}, \mathrm{Nb}$ and Ta may form complexes with a variety of ligands such as nonbridging oxygen (NBO), F, B, etc. (Keppler, 1993 and Bau, 1996). The stability of these complexes is constrained by the charge and ionic radius of individual elements. The lanthanide tetrad effect is accompanied by the behavior of non-charge and radius controlled (nonCHARAC) elements. The more pronounced the tetrad effect, the greater the deviation is for the diadochic elements from the CHARAC behavior (Jahn et al., 2001).

The REEs tetrad effect is most visible in late magmatic differentiates with strong hydrothermal interactions or deuteric alteration. This includes highly evolved leucogranites, pegmatites and mineralized granites. The fractionation of elements which are similar to each other in term of ionic radius and charge is regarded to be sensitive to changes in melt composition during magma differentiation (Bau, 1996, 1997; Irber et al., 1997).

The tetrad effect values are plotted vs. the $\mathrm{K} / \mathrm{Rb}, \mathrm{Sr} / \mathrm{Eu}, \mathrm{Eu} / \mathrm{Eu}^{*}, \mathrm{Y} / \mathrm{Ho}$ and $\mathrm{Zr} / \mathrm{Hf}$ ratios (Fig. 8) to search for common underlying processes in trace element behavior.

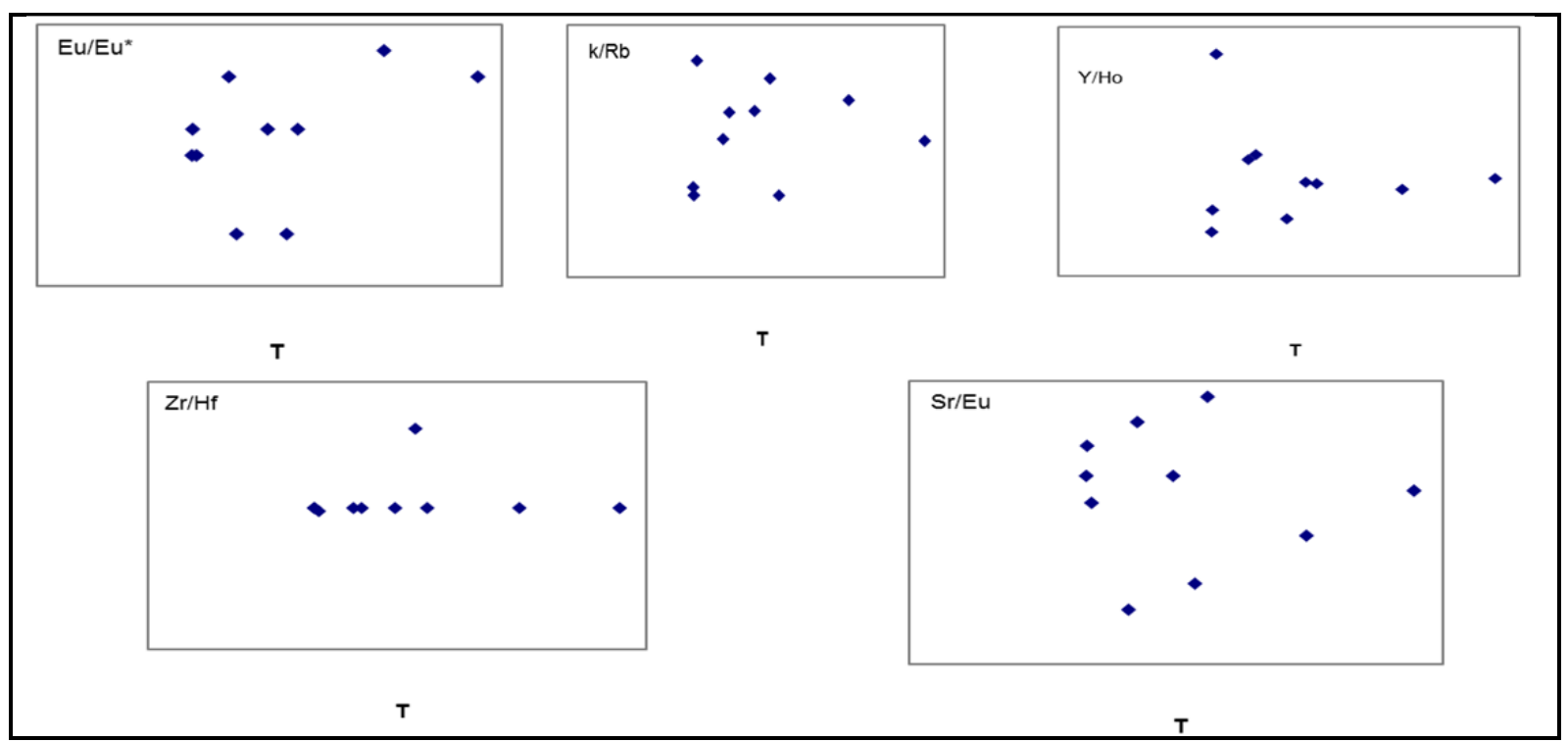

Fig. 8: Tetrad effect (T) vs. Eu/Eu*, $\mathrm{K} / \mathrm{Rb}, \mathrm{Y} / \mathrm{Ho}, \mathrm{Zr} / \mathrm{Hf}$ and $\mathrm{Sr} / \mathrm{Eu}$ ratios in mineralized pegmatites

The chondritic $\mathbf{K} / \mathbf{R b}$ ratio is 242 (Anders and Grevesse, 1989), and the average for magmatic rocks is 230 (Taylor, 1965). With increasing degree of differentiation, $\mathrm{Rb}$ fractionates preferentially into the residual melt and the $\mathrm{K} / \mathrm{Rb}$ ratios decrease in highly evolved magmatic systems below 50 . In the studied mineralized pegmatites, the $\mathrm{K} / \mathrm{Rb}$ ratio ranges from 40 to 240 with an average of 92.2 . It is lowered in comparison with the average in non-mineralized pegmatites (145). The tetrad effect is weakly correlated with the $\mathrm{K} / \mathrm{Rb}$ (Fig. 8). $\mathrm{K} / \mathrm{Rb}$ is commonly used to characterize the evolution of granitic melts. Ratio values less than 100 are regarded to indicate the interaction with an aqueous fluid phase (Clarke, 1992) or mineral growth in the presence of aqueous fluids (Shearer et al., 1985). 


\section{Geochemical contributions of Ree's bearing pegmatites}

The chondritic ratio of $\mathrm{Y} / \mathrm{HO}$ is 28 (Anders and Grevesse, 1989). In the studied non-mineralized pegmatites, the average Y/Ho ratio is 100.2 while it is very low (19) in the mineralized pegmatites and negatively correlated with the tetrad effect (Fig. 8). The Y/Ho ratio was proposed as a factor to identify non-charge and non-ionic size controlled magmatic trace element behavior such as found in aqueous systems (Bau, 1996). The fractionation behavior of highly charged ions, which form strong chemical complexes, is additionally influenced by their electron configuration and the character of chemical bonding between a central ion and a ligand. Bau and Dulski (1995) suggested the complexation with fluorine as major cause for values $>28$. The $\mathrm{F}$ content in kadabora granites and the pegmatites is $1520 \mathrm{ppm}$ and 1780 ppm respectively (Abdalla and El Afandy, 2003).

$\mathrm{The} \mathrm{Zr} / \mathrm{Hf}$ values in granites average is 39 (Erlank et al., 1978). The chondritic ratio is 38 (Anders and Grevesse, 1989). The average for pegmatites is at about 25 (Erlank et al., 1978). $\mathrm{Zr} / \mathrm{Hf}$ ratio decreases with increasing evolution of the silicate melt. In the studied non-mineralized pegmatites, the $\mathrm{Zr} / \mathrm{Hf}$ value is 43. It is decreased to 3.8 for the mineralized pegmatites during the crystallization of the kadabora granites.

$\mathrm{Sr} / \mathrm{Eu}$ ratio displays a distinctive behavior during magma evolution and bears information relevand to trace element behavior in general. The chondritic $\mathrm{Sr} / \mathrm{Eu}$ value is 139 (Anders and Grevesse, 1989). Sr/Eu value in the mineralized pegmatites ranges from 6.8 to 33 with an average of 21.1 while for the nonmineralized ones it is ranging from 35 to 180 with an average of 89.4. Sr/Eu ratio negatively correlated with the tetrad effect (Fig. 8).

$\mathrm{Eu} / \mathrm{Eu}$ * ratio is correlated with the tetrad effect (Fig. 9). The primary cause of the tetrad effect, i.e., magma-fluid interaction, has not only depleted $\mathrm{Eu}$ in the rock, but also resulted in unusual negative Eu anomalies in all constituent minerals including K-feldspars (Zhao et al., 1999).

Fig. 9: Enrichmentdepletion diagram for trace elements in mineralized relative to non-mineralized pegmatites.

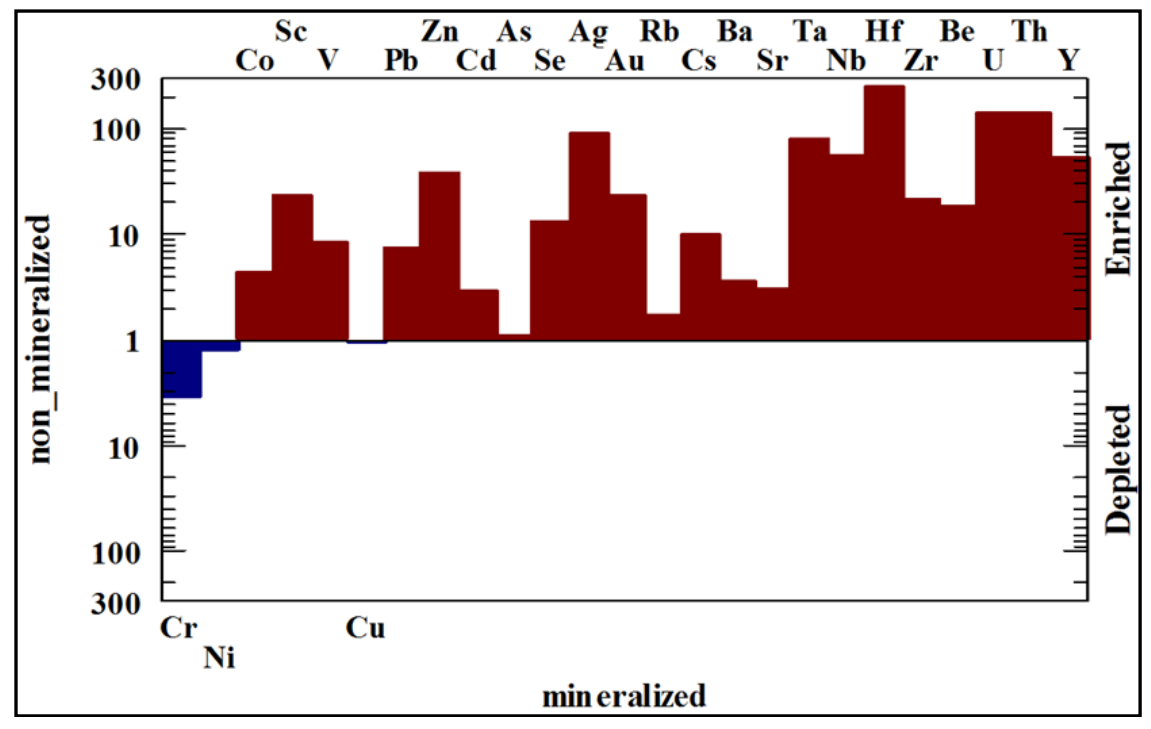

The regional compositional differences of pegmatites may, of course, reflect the original composition of the magmas from which they were derived, but the reasons for such compositional differences are unexplained. Another curious fact is that only relatively few pegmatites carry the unusual elements; over $90 \%$ consist essentially of quartz and feldspar and are quite barren of rare minerals. Many geologists who have studied the complex pegmatites believe that the unusual minerals were deposited during one or more phases of hydrothermal replacement following the original formation of a simple quartz -feldspar-mica pegmatite. The idea helps to explain the often erratic occurrence of a single rare-mineral pegmatite among many simple ones (Mason, 1966).

The parent magma for the studied mineralized pegmatites have undergone extensive magmatic differentiation, during which intense interaction of the residual melt with aqueous hydrothermal fluids most probably rich in $\mathrm{F}$ (as indicated from the presence of fluorite in the rocks) resulted in the tetrad effect of REEs distribution and formation of highly mineralized pegmatites. 
El Afandy, et al

\section{Distribution of trace elements}

The Clark Concentration (CC) was calculated for estimation the enrichment of the trace elements in the mineralized relative to the non-mineralized pegmatites. $\mathrm{CC}$ values were arranged in decreasing order show the following geochemical spectrum:

(Hf, REEs, U, Th, Ag, Ta, Nb, Y, Zn, Au, Sc, Zr, Be, Se, Cs, Pb, Co, V, Ba, Sr, Cd, Rb, As, Cu, Ni, Cr), (Fig. 9).

The assemblage of accessory minerals encountered in the mineralized pegmatites is zircon, allanite, fergusonite, uranothorite, betafite, samarskite, pyrochlore, bastnaesite, cerianite, rutile, euxenite and opaques (Dawood, 1999; Abdalla and El Afandy, 2003; Ahmed, 2004). The nature of such minerals is reflected in the trace elements contents as follows: zircon ( $\mathrm{Zr}, \mathrm{Hf}, \mathrm{Th})$; fergusonite $(\mathrm{U}, \mathrm{Th}, \mathrm{Zr})$; uranothorite $(\mathrm{Th}, \mathrm{U})$; betafite $(\mathrm{U}, \mathrm{Nb}, \mathrm{Ta})$; samarskite $(\mathrm{Nb}, \mathrm{Ta}, \mathrm{U})$; pyrochlore $(\mathrm{Nb}, \mathrm{Ta})$; bastnaesite (REEs); cerianite (Ce, $\mathrm{Th}, \mathrm{Nb}, \mathrm{Ta}, \mathrm{Zr}$ ) and rutile (Ti).

The geochemical spectrum of rare earth elements is as follows:

(Lu, Yb, Tm, Er, Ho, Dy, Ce, Pr, Tb, Sm, La, Gd, Nd, Eu)

The REEs pattern of the mineralized pegmatites reflects the nature of accessory minerals where they are acting as REEs accumulators e. g. uranothorite, betafite, bastnaesite and cerianite host LREEs meanwhile zircon, allanite and samarskite host HREEs.

Pegmatites often contain minor amounts of sulfides and arsenides. Gold has been recorded from some. They show many of the characteristics of hydrothermal veins, and examples of pegmatites passing into sulfide-bearing quartz veins have been described (Mason, 1966).

Gold content in the studied mineralized pegmatites is extraordinary; it ranges from $6 \mathrm{ppm}$ to $47 \mathrm{ppm}$ with an average of $25.2 \mathrm{ppm}$. Moreover, the mineralized pegmatites are highly enriched in $\mathrm{Ag}, \mathrm{Zn}, \mathrm{Se}, \mathrm{Pb}$ and Co compared to the non-mineralized ones. Such enrichment indicates the presence of gold associated REEs mineralization.

\section{CONCLUSIONS}

Pegmatitic bodies encountered within the Kadabora pluton to which they are spatially and genetically related differentiated into REEs, $\mathrm{U}, \mathrm{Th}, \mathrm{Y}, \mathrm{Zr}, \mathrm{Hf}, \mathrm{Nb}, \mathrm{Ta}$ mineralized and normal non-mineralized.

The mineralized pegmatites are characterized by high $\Sigma$ REEs content ranging from $3404 \mathrm{ppm}$ to 15196 with an average of $7516 \mathrm{ppm}$. The REEs pattern is characterized by HREEs enrichment relative to LREEs with convex tetrad effect and a sharp negative Eu anomaly. This pattern is well demonstrated in highly differentiated rocks with strong hydrothermal interaction.

The parent magma for the mineralized pegmatites have undergone extensive magmatic differentiation, during which intense interaction of the residual melt with aqueous hydrothermal fluids resulted in the tetrad effect of REEs distribution and formation of highly mineralized pegmatites.

The non-mineralized pegmatites characterized by low contents of $U$ (3.8-20.8ppm) and Th (5.4-28 $\mathrm{ppm}$ ), with $\mathrm{Th} / \mathrm{U}$ ratio in the range from 1.3 to 3.1 and 1.8 in average, while the mineralized pegmatites are characterized by high contents of $\mathrm{U}(192-2520 \mathrm{ppm})$ and $\mathrm{Th}(600-4340 \mathrm{ppm})$, with $\mathrm{Th} / \mathrm{U}$ ratio in the range from 1.3 to 3.2 and 1.8 in average. The mineralized pegmatites are enriched in $U$ and $T h$ by an enrichment ratio of more than 180 , compared to the non-mineralized pegmatites.

The radioactive bearing minerals identified by XRD and ESEM techniques are zircon, fergusonite, uranothorite, betafite, and samarskilte.

The constant $\mathrm{Th} / \mathrm{U}$ ratio in both mineralized and non-mineralized pegmatites and the positive correlation between $\mathrm{U}$ and $\mathrm{Th}, \mathrm{Zr}$, Ta, Nb, Hf and $\Sigma$ REEs in the studied pegmatites reflect that the enrichment of uranium and thorium is related to magmatic (hypogene) processes. Also, the enrichment of 


\section{Geochemical contributions of Ree's bearing pegmatites}

both uranium and/or thorium bearing minerals (zircon, fergusonite, uranothorite, betafite, and samarskilte) at the residual magma (pegmatite) indicates that, the enrichment is due to magmatic processes.

\section{REFERENCES}

Abdalla, H. M. and El Afandy, A. H. (2003): Contrasting mineralogical and geochemical characteristics of two A-type pegmatite fields, Eastern Desert, Egypt. Egypt. Mineral. 15, 287-328.

Abd El Wahed, A. A., Raslan, M. F. and El Husseiny, M. O. (2005): Radioactive pegmatites of umm lassifa granitic pluton, central eastern desert, egypt: mineralogical investigation. The $9^{\text {th }}$ International Mining, Petrolium and Metallurgical Engineering conference Cairo Univ.

Abu El-Enen, M. M. (1995): Geological, geochemical and mineralogical studies on the metamorphic rocks between Wadi Um-Maghra and Wadi Tweiba area, Southeastern Sinai, Egypt. Ph.D. Thesis, ElMansoura Univ., Egypt.

Abu Steet, A. A, El Sundoly,H. I. and Abdel Hamid, A. A. (2018):Rare elements distribution and mineralization potentiality of pegmatites in gabal abu samyuk granite, north eastern desert, Egypt. Egy. J. Geo., v. 62, p.

Ahmed, A. A. (2004): Geology, petrography, geochemistry and radioactivity of Kadabora area, Central Eastern Desert of Egypt. Ph. D. Thesis, South Valley Univ., Egypt.

Anders E. and Grevesse N. (1989): Abundances of the elements: Meteoritic and solar. Geochim. Cosmochim. Acta 53, 197-214.

Bau, M., (1996): Controls on the fractionation of isovalent trace elements in magmatic and aqueous systems: evidence from $\mathrm{Y} / \mathrm{Ho}, \mathrm{Zr} / \mathrm{Hf}$ and lanthanide tetrad effect. Contrib. Mineral. Petrol. 123, 323333.

Bau, M. (1997): The lanthanide tetrad effect in highly evolved felsic igneous rocks-A reply to the comment by Y. Pan. Contrib. Mineral. Petrol. 128, 409-412.

Bau, M. and Dulski, P. (1995): Comparative study of ytrium and rare-earth elements behaviors in fluorinerich hydrothermal fluids. Contrib. Mineral. Petrol. 119, 213-223.

Brotzen, O. (1959): Outline of mineralization in zoned granitic pegmatites, a qualitative and comparative study. Geol. Foren. Stockholm Forh., bd. 81, h. 2, No. 496. 1-98.

Cerny. P. (1982): Petrogenesis of granitic pegmatites. In: P. Cerny (ed). Granitic pegmatites in science and industry, Mineral Assoc. Canada, short course Hand Book 8. 405-461.

Cerny, P. and Burt, D. M. (1984): Paragenesis crystalls-chemical characteristics and geochemical evolution of micas in granite pegmatites In : S. W. Bacley (ed) Mica, Mineral Soc. Am. Reu. Mineral. 13, 257-297.

Clarke D. B. (1992): The mineralogy of peraluminous granites: A review. Can. Mineral. 19, 3-17.

Cocherie A., Johan V., Rossi P., and Stemprok M. (1991): Trace element variations and lanthanide tetrad effect studied in a Variscan lithium albite granite: Case of the Cinovec granite (Czechoslovakia) [abstract]. In Source. Transport and Deposition of Metals: Proceedings of an SGA Anniversary Meeting (eds. M. Pagel and J. L. Leroy), pp. 745-749. Balkema.

Darwish, N. K. (1991): Geological studies on granitic and pegmatitic rocks South West Taba, Sinai, Egypt. M. Sc. Thesis, El Mansoura Univ., Egypt.

Dawood, N. A. M. (1999): Geochemical and mineralogical studies of younger granites and uraniferous pegmatites at Gebel Abu Dob, Central Eastern Desert, Egypt. Ph. D. Thesis, Mansoura Univ., Egypt.

Draz, M. O. (2013): Petrography and radioactivity of the feldspar veins and pegmatite bodies, South Gebel Um Anab, North Eastern Desert, Egypt. The $10^{\text {th }}$ Conf. Geochem., Alex. Univ. (abstracts)

El-Aassy, I. E., Botros, N. H., Ibrahim, M. E., Hammad, M. S. and Hassan, M. A. (1993): A new beryl occurrence in Sinai, Egypt. Min. Soc. Egypt 5:139-151.

El-Aassy, I. E., Shazly, A. G., Hussien, H. A., Heikal, M. T. S. and El-Galy, M. M. (1997): Pegmatite of Nuweiba-Dahab area, West Gulf of Aqaba, Sinai, Egypt: Field aspects, mineralogy, geochemistry and radioactivity. The $3^{\text {rd }}$ Conf. Geochem., Alex. Univ. 3:139-151.

El-Essawy, M. A., El-Metwally, A. A., Katta, L. A. and Darwish, N. K. (1997): Younger granites and pegmatites from Taba area, Southeastern Sinai, Egypt. Egypt. J. Geol. 41(1):495-518. 
El Afandy, et al

El-Mowafy, A. A., Moussa, E. M. M. and El-Galy, M. M. (2001): Younger granites and pegmatites around Wadi Sabbagh-Wadi Haleifiya area, South-east Sinai, Egypt: Their petrogenesis and U-Th distribution. $5^{\text {th }}$ Inter. Conf. Geochem. Alex. Univ., Egypt 5:227-250.

El-Nahas, H. A. (2012): Mineralogy and radioactivity of some pegmatite bodies, southeastern Sinai, Egypt. Nuclear sciences scientific J. 1:57-68.

El-Sharkawy, M. F. and Harraz, H. Z. (2001): Progressive boron metasomatism next to a pegmatite at Wadi Sikait area, South Eastern Desert, Egypt. $5^{\text {th }}$ Intern. Conf. Geochem. Alex. Univ., Egypt 5 25-51.

El-Shazly, E. M., El-Ramly, M. F., Saleeb, G. S. and Rasmy, A. H. (1975a): Geology, petrogenesis and mode of formation of asbestos-vermiculite deposits at Hafafit, Egypt. J. Geol. 19:87-104.

El-Shazly, E. M., El-Ramly, M. F., Saleeb, G. S. and Rasmy, A. H. (1975b): Geology, petrogenesis and mode of formation of corundum deposits at Hafafit, Egypt. J. Geol. 19:125-152.

El Sheshtawy, Y. A., Aly, M. M. and Ahmed, A. M. (1988): Geochemistry and tectonic environments of the granite-pegmatite dykes around Wadi El-Morakh area, Sinai, Egypt. Mansoura Sci. Bull. 15(1):5581.

Erlank A. J., Marchant J. W., Cardoso M. P., and Ahrens L. H. (1978): Zirconium. In Handbook of Geochemistry (ed. K. H. Wedepohl) Vol 11/4, pp 40 B-O. Springer.

Fritz. H., Abdelsalam, M., Ali, K. A., Bingen, B., Collins, A. S., Fowler, A. R., Ghebreab, W., Hauzenberger, C. A., Johnson, P. R., Kusky, T. M., Macey, P., Muhongo, S., Stern, R. J. and Viola, G. (2013): Orogen styles in the East African Orogen: a review of the Neoproterozoic to Cambrian tectonic evolution. J. Afr. Earth Sci. 86:65-106.

Gabelman, R., (1970): Speculations on the uranium ore fluid. In: Uranium Exploration geology, 315-330.

Ghazaly, M. K, El Afandy, A. H. Fawzy, Kh. M. and Fahmy, M. A. (2013): The pegmatitic rocks of ElHudi and Um Hibal areas, Southeastern Desert, Egypt: genesis and petrological characteristics. Arab. J. Geosci, DOI 10.1007/s12517-013-1168-y.

Goldschmidt, V.M., (1954): Geochemistry. Oxford: University Press. 730p. Oxford

Grinsburg, A. I., Timofeyer, I. N. and Feldman, L. G. (1979): Principales of geology of the granitic pegmatites. Nadra Moscow, 296p. in Russian.

Hermann, A. G., (1970): Yittrium and lanthindes, in Wedepohl, K.H., ed., Handbook of geochemistry, Berlin, Springer, 2, 59-71.

Irber W., Förster H.-J., Hecht L., Möller P., and Morteani G. (1997): Experimental, geochemical, mineralogical and O-isotope constraints on the late-magmatic history of the Fichtelgebirge granites (Germany). Geol. Rdsch. 86 (suppl.), 110-124.

Irber, W. (1999): The lanthanide tetrad effect and its correlation with $\mathrm{K} / \mathrm{Rb}, \mathrm{Eu} / \mathrm{Eu}, \mathrm{Sr} / \mathrm{Eu}, \mathrm{Y} / \mathrm{Ho}$ and $\mathrm{Zr} / \mathrm{Hf}$ of evloving per-aluminous granite suites. Geochim. Cosmochim. Acta 63, 489-508.

Jahn, B, Wu, F., Capdevila, R., Martineau, F, Zhao, Z. and Wang, Y. (2001): Highly evolved juvenile granites with tetrad REE patterns: the Woduhe and Baerzhe granites from the Great Xing'an Mountains in NE China. Lithos, 59, 171-198.

Jahns, R. H. and Burnham, C. W. (1969): Experimental studies of pegmatite genesis, I, A model for the derivation and crystallization of granitic pegmatites: Econ. Geology, vol. no. 8, p. 843-864.

Kawabe, I. (1995): Tetrad effects and fine structures of REE abundance patterns of granitic and rhyolitic rocks: ICP-AES determinations of REE and Y in eight GSJ reference rocks. Geochem. J. 29, 213-230.

Keppler, H. (1993): Influence of flurine on the enrichment of high field strength trace elements in granitic rocks. Contrib. mineral. Petrol. 114, 479-488.

Mason, B. (1966): Principles of geochemistry $3^{\text {rd }}$ Ed. John Wiley, New York, 310 P.

Masuda, A., Kawakami, O., Dohomoto, Y, and Takemaka, T. (1987): Lanthanide tetrad effects in nature two multually opposite type W and M. Geochem. J. 21, 119-124.

McLennan S. M. (1994): Rare earth element geochemistry and the "tetrad" effect. Geochim. Cosmochim. Acta 58, 2025-2033.

Möller P. and Muecke G. K. (1984): Significance of europium anomalies in silicate melts and crystal-melt eqilibria; a re-evaluation. Contrib. Mineral Perol., 87. 242-250.

Monecke, T., Kempe, U, Monecke, J., Sala, M and Wolf, D. (2002): Tetrad effect in rare earth element distribution patterns: A method of quantification with application to rock and mineral samples from granite related rare metal deposits. Geochimica et Cosmochimica Acta, 66(7), 1185-1196. 


\section{Geochemical contributions of Ree's bearing pegmatites}

Muecke G. K. and Clarke D. B. (1981): Geochemical evolution of the South Mountain Batholith, Nova Scotia: rare-earth element evidence. Can. Mineral. 19, 133-145.

Norton, J. J. (1983): Sequence of mineral assemblages in differentiated granitic pegmatites, Econ. Geol., $78,854-874$.

O'Connor, P.J., Buch, P.M. and Williams, C.T., (1982): Abundance and distribution of uranium and thorium in the northern units of Leinster granites, Ireland. Geol. Mag., 119(6), 581-592.

Proctor, P. D. and El Etr, H. A. (1968): Layered pegmatites, southern Wind River Mountains, Fremont Country. Wyoming, Econ. Geol. 63, 595-611.

Rashwan, A. A. (1991): Petrography, geochemistry and petrogenesis of the Migif-Hafafit gneisses at Hafafit mine area, South Eastern Desert, Egypt. Sci. Ser. Intern. Barea 5: Forschangszentran Julich $\mathrm{GmbH}$

Rogers, J. J. and Adams T. S. (1969): Uranium In: Wedepohl, K. H. (ed) Handbook of geochemistry New York Springer-verlag, V-4: 92 bt to 92 C10.

Shearer C. K., Papike J. J., and Laul J. C. (1985): Chemistry of potassium feldspars from three zoned pegmatites, Black Hills, South Dakota: Implications concerning pegmatite evolution. Geochim. Cosmochim. Acta, 49, 663-673.

Sherif, H. M. Y. (2003): Primary uranium mineralization of Wadi El-Beida granite pegmatite, south Sinai, Egypt. Egypt. J. Geo. 47(1):175-188.

Stewart, D. B. (1978): Petrogenesis of lithium rich pegmatites. Amen mineral, 63, 970-980.

Sun, S.S., and McDonough, W. F., (1989): Chemical and isotopic systematic of ocean basalts Implications for mantle composition and processes, In: Saunders, A.D., and Norry, M.J. (eds) Magmatism in the Ocean Basins, Geol. Soc. London, Spec. Publ. 42, 313-345.

Taylor S. R. (1965): The application of trace element data to problems in petrology. Phys. Chem. Earth 6, 133-213.

Zhao, Z. H., Xiong, X. L. and Han, X. D. (1999): The formation mechanism of REE tetrad in granites. Sci. China, Ser, D: Earth Sci. 29, 331- 338 (In Chinese). 
El Afandy, et al

\section{إسهامات جيوكيميائية في صخور البجماتيت الحاملة للعناصر الأرضية النادرة فى متذاخل كادابورا الجرانيتى ، وسط المبط ،

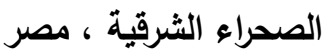 \\ عادل حسن الأفندى و ناصر مصطفي مغازى و جيهان بكر الثايب \\ الخلاصة}

تتواجد الأجسام البجمانتية داخل جرانيت كادابورا الذى تتنمي إليه من حيث المكان والنشأة ـ هذه الأجسام حاملة للعناصر الأرضية النادرة وكل من اليورانيوم والثوريوم والإيتريوم والزركونيوم والهافنيوم والنيوبيوم والنتنالم بالإضافة للسيزيوم • يتميز

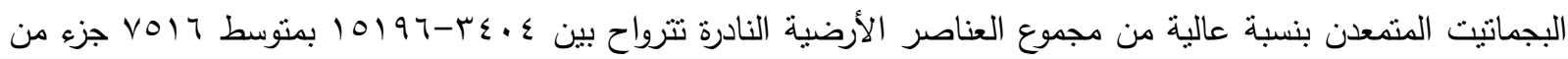
المليون • وقد تم رصد صحبة من المعادن الإضافية في البجماتيت المتمعدن ممثلة بالزيركون، الألانيت ، فريجسونيت ، يورانينيت ، بيتافيت ، سمارسكايت ، بيروكلور، باستانسيت ، سيرانيت ، روتيل ، ليكوجسن والمعادن المعتمة ـ كل من هذه

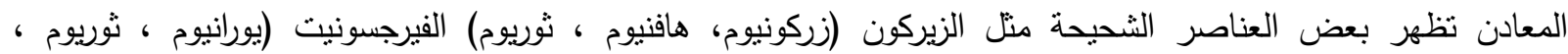

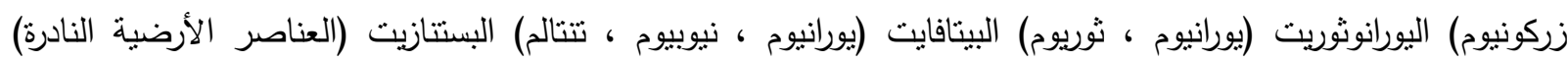

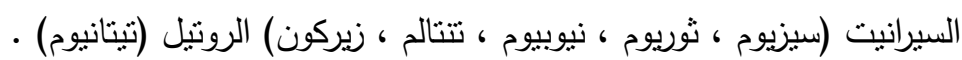
يعكس نموذج نوزيع العناصر الأرضية النادرة فى البجماتيت المتمعدن دور هذه المعادن كمتراكمات للعناصر الأرضية النادرة مثل : البيتافايت ، البستانيزيت والسيريانيت كعائل للعناصر الأرضية النادرة الخفيفة بينما الزيركون والسمارسكايت كعائل

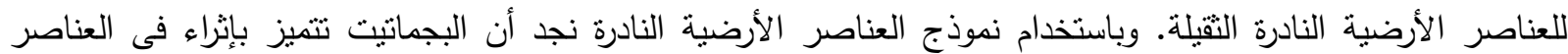
الأرضية العالية مقارنة بمثيلتها المنخفضة مع تأثثر رباعي محدب وشاذة ايريبيوم سالبة عالية .

هذا النمط التوضيحي يعكس صخور عالية التفارق مصحوبة بتفاعل حارمائى ذاتي قوى • يبدو ان المجما الأم قد تعرضت

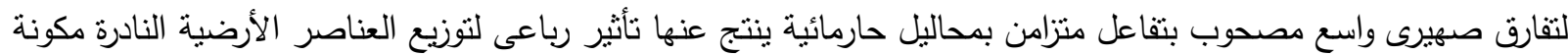

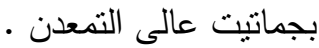

